PREPARED FOR SUBMISSION TO JCAP

\title{
Using the redshift evolution of the Lyman- $\alpha$ effective opacity as a probe of dark matter models
}

\section{Anjan Kumar Sarkar ${ }^{a}$ Kanhaiya L. Pandey ${ }^{b}$ Shiv K. Sethi $^{a}$}

\author{
${ }^{a}$ Raman Research Institute, Sadashivnagar, Bangalore, Karnataka 560080 \\ ${ }^{b}$ International Center for Theoretical Sciences, TIFR, Shivakote, Hesaraghatta, Ban- \\ galore, Karnataka 560089 \\ E-mail: anjans@rri.res.in, kanhaiya.pandey@icts.res.in, sethi@rri.res.in
}

\begin{abstract}
Lyman- $\alpha$ forest data are known to be a good probe of the small scale matter power. In this paper, we explore the redshift evolution of the observable effective optical depth $\tau_{\text {eff }}(z)$ from the Lyman- $\alpha$ data as a discriminator between dark matter models that differ from the $\Lambda$ CDM model on small scales. We consider the thermal warm dark matter (WDM) and the ultra-light axion (ULA) models for the following set of parameters: the mass of ULA, $m_{a} \simeq 10^{-24}-5 \times 10^{-22} \mathrm{eV}$ and WDM mass, $m_{\mathrm{wdm}}=0.1-4.6 \mathrm{keV}$. We simulate the line-of-sight HI density and velocity fields using semi-analytic methods. The simulated effective optical depth for the alternative dark matter models diverges from the $\Lambda$ CDM model for $z \gtrsim 3$, which provides a meaningful probe of the matter power at small scales. Using likelihood analysis, we compare the simulated data with the high-resolution Lyman- $\alpha$ forest data in the redshift range $2<z<4.2$. The analysis yields the following $1 \sigma$ bounds on dark matter masses: $m_{\mathrm{wdm}}>0.7 \mathrm{keV}$ and $m_{\mathrm{a}}>2 \times 10^{-23} \mathrm{eV}$. To further test the efficacy of our proposed method, we simulate synthetic data sets compatible with the $\Lambda$ CDM model in the redshift range $2 \leq z \leq 6.5$ and compare with theory. The $1 \sigma$ bounds obtained are significantly tighter: $m_{\mathrm{wdm}}>1.5 \mathrm{keV}$ and $m_{\mathrm{a}}>7 \times 10^{-23} \mathrm{eV}$. Although our method provides an alternative way of constraining dark matter models, we note that these bounds are weaker than those obtained by high-resolution hydrodynamical simulations.
\end{abstract}




\section{Contents}

1 Introduction $\quad 1$

2 Alternative dark matter models: warm dark matter and ultra-light axions

3 Simulating line-of-sight HI density fluctuations: Lyman- $\alpha$ clouds

4 Neutral hydrogen and the Lyman- $\alpha$ optical depth $\quad 7$

4.1 Data for $\tau_{\text {eff }} \quad 8$

5 Results $\quad \mathbf{9}$

5.1 Effective optical depth and dark matter models 10

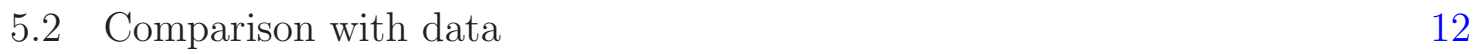

5.3 Comparison with simulated data $\quad 15$

6 Summary and Conclusions $\quad 16$

$\begin{array}{llr}7 & \text { Acknowledgment } & 19\end{array}$

\section{Introduction}

The standard cosmological model has proved to be spectacularly successful during the past three decade. Among other probes, the measurement of CMB temperature and polarization anisotropies, galaxy clustering as revealed by large surveys, and the detection of high-redshift supernova 1a have been key to this success [1-7]. An important ingradient of the concordance $\Lambda \mathrm{CDM}$ model is the cold dark matter. However, even after extensitve laboratory and astronomical searches, the nature of dark matter is yet to be directly determined. Its properties are indirectly inferred based on many observations covering a wide range of length scales and epochs of the universe (e.g. $[2-4,8])$.

In the $\Lambda$ CDM model, the cold dark matter particle corresponds to the Weakly Interacting Massive Particle (WIMP), which in turn is inspired by the well-known WIMP miracle[9]. The supersymmetric extension of the standard model of particle physics is consistent with a particle with self-annihilation cross-section $\langle\sigma v\rangle \sim 3 \times$ $10^{-26} \mathrm{~cm}^{3} \mathrm{~s}^{-1}$ and mass in the range $100-1000 \mathrm{GeV}$. This theory correctly predicts the dark matter abundance inferred by the cosmological observations. This coincidence has spurred many direct [10-13], indirect[14-16] and collider [17, 18] searches of the WIMP worldwide. However, none of these experiments have yet succeeded in providing consistent information about the particle nature of the dark matter.

While CMB and galaxy clustering observations show that the CDM is a good candidate of dark matter for scales $k<0.1 \mathrm{Mpc}^{-1}$, there exist long-standing astrophysical 
issue with the model at smaller scales. N-body simulations based on the CDM model predict an order of magnitude larger number of satellite galaxy of the Milky way as compared to the observations [19-22]. CDM N-body simulations predict a cuspy profile at the center of galaxies but the observed profile is flat [23, 24]. Another issue to emerge from the comparison of N-body simulation with observations is the "too big to fail" problem $[25,26]$. All these issues provide a motivation to go beyond the standard CDM paradigm and to consider alternatives, which differ from the CDM on galactic scales but reproduce its success on the cosmological scales.

The Lyman- $\alpha$ forest are the observed absorption features along the directions of QSOs owing to the fluctuations in the neutral hydrogen (HI) density of the predominantly ionized diffuse IGM in the post-reionization era $(z \leq 6)$. Hydrodynamical simulations have shown these fluctuations correspond to mildly non-linear density contrast $(\delta<10)$ of the underlying density field (e.g. [27-31] and references therein). As this allows one to probe the fluctuations of the density field at scales comparable to the Jeans' scale of the IGM ( $\left.k \simeq 5-7 \mathrm{Mpc}^{-1}\right)$ in the redshift range $2<z<5$, the Lyman- $\alpha$ forest provide a suitable setting for the measurement of the matter power spectrum for a vast range of scales including small scales not accessible to other probes such as the galaxy surveys[27-31]. These data have found widespread applications in cosmology, e.g. the measurement of bispectrum [32, 33], the estimation of the cosmological parameters [34-36], obtaining constraints on the neutrino mass [37, 38], dark energy [39], and the detection of the baryon Acoustic Oscillations (BAO) [40-42].

In this paper we propose a novel method to investigate alternative dark matter models using Lyman- $\alpha$ forest data. These data allow us to measure the flux decrement as a function of redshift, which can be quantified in terms of the Lyman- $\alpha$ effective optical depth $\tau_{\text {eff }}(z)$. The observational data have determined $\tau_{\text {eff }}$ over the redshift range $2 \leq z \leq 5$ for a range of spectral resolutions [43-46]. For comparison with the data, we simulate the one-dimensional HI density and velocity fields using semianalytic methods (e.g. [47]) to compute the effective optical depth in the redshift range of interest. The redshift evolution of the theoretically estimated effective optical depth is compared with the Lyman- $\alpha$ data. We explore whether this method can be used to discriminate between dark matter models with different small-scale matter power (e.g. [48]). We also compare theoretical predictions with simulated data.

We study two alternative dark matter models for comparison with the Lyman- $\alpha$ data. One of these models is the warm dark matter (WDM) model, which has been extensively studied for cosmological applications [49-53]. In the model we consider, the dark matter particle is coupled to the thermal bath in the early universe and its mass lies in the range from a few hundred eVs to $10 \mathrm{keV}$. Thermally-produced gravitinos and the sterile neutrino are the few possible candidates for the WDM particles $[54,55]$. The ultra-light axions (ULA) arise naturally within the framework of axiverse [56]. The ULA have masses in the range $10^{-33}<m_{a}<10^{-20} \mathrm{eV}$ and behave like a coherent scalar field. For both the WDM and the ULA models, the matter power is suppressed at small scales and these models have been studied for various astrophysical and cosmological applications ([53, 57-76]). These models have also been studied using Lyman- $\alpha$ forest data for constraining small-scale power $([62,63,77-79])$. We also discuss how our 
results compare with these findings.

In the following section, we briefly discuss the two alternative dark matter models we study. In section 3, the semi-analytic simulations are described in detail. In section 4 we discuss the observables and the data. In section 5 , we present our main results. In section 6, we summarize the main findings and conclude.

Throughout this paper, we use the following cosmological parameters: $\Omega_{c 0}=$ $0.2285, \Omega_{b 0}=0.046, \Omega_{\nu 0}=0.0013, \Omega_{k 0}=0, \Omega_{\Lambda 0}=0.7242$ and $h=0.7[3]$.

\section{Alternative dark matter models: warm dark matter and ultra-light axions}

In this section, we briefly discuss the two alternative dark matter models we consider in our study. They are both motivated by the observational evidence that while the usual $\Lambda$ CDM model is in excellent agreement with CMB and galaxy clustering at at $k \lesssim 0.2 \mathrm{Mpc}^{-1}$, it overpredicts the matter power at smaller scales. Both the warm dark matter (WDM) the ultra-light axion (ULA) models yield diminished matter power at small scales.

In the WDM scenario we consider, the dark matter particles are lighter than the cold dark matter particles and are coupled to the thermal bath at early times. When the temperature of the universe is much larger than the mass of the dark matter particle, $T \gtrsim m_{\mathrm{wdm}}$, the particles are relativistic and free-stream, which causes suppression of the matter power at scale smaller than horizon size corresponding to the time at which $T \simeq m_{\text {wdm }}$ (e.g. [80-84]. At later times, the dark matter particles become nonrelativistic and their velocity dispersion falls as $1 / a$, which causes them to behave as the CDM particles at late times. The free-streaming length scale $k_{\mathrm{fs}}$ is given by $[85,86]$ :

$$
k_{\mathrm{fs}} \simeq\left(\frac{0.3}{\Omega_{\mathrm{wdm}}}\right)^{0.15}\left(\frac{m_{\mathrm{wdm}}}{\mathrm{keV}}\right)^{1.15} \mathrm{Mpc}^{-1}
$$

where $\Omega_{\mathrm{wdm}}$ gives the WDM matter density expressed in the units of the critical mass density of the universe. For $m_{\mathrm{wdm}}=0.3 \mathrm{keV}$, the free-streaming length scale, $k_{\mathrm{fs}} \simeq$ $0.3 \mathrm{Mpc}^{-1}$.

The impact of the free-streaming of the WDM particles on the matter power spectrum can be quantified through the transfer function $T(k)[86,87]$ :

$$
T(k)=\left[\frac{P_{\mathrm{WDM}}(k)}{P_{\mathrm{CDM}}(k)}\right]^{1 / 2}=\left[1+(\alpha k)^{2 \mu}\right]^{-5 / \mu},
$$

Here $P_{\mathrm{WDM}}(k)$ and $P_{\mathrm{CDM}}(k)$ give the linear matter power spectrum for the WDM and CDM model, respectively and $\alpha, \mu$ are the parameters that are used to model the transfer function $T(k)$ for the WDM model. We use the following fit for the parameters of the transfer function $\mu$ and $\alpha$ [81]: $\mu=1.12$ and

$$
\alpha=0.049\left[\frac{m_{\mathrm{wdm}}}{\mathrm{keV}}\right]^{-1.11}\left[\frac{\Omega_{\mathrm{wdm}}}{0.25}\right]^{0.11}\left[\frac{h}{0.7}\right]^{1.22} h^{-1} \mathrm{Mpc}
$$


It follows from Eqs. (2.2) and (2.3) that as the $m_{\mathrm{wdm}}$ is decreased, the matter power is erased at progressively larger scales. In this work we consider WDM particles in the mass range: $0.1 \mathrm{keV}<m_{\mathrm{wdm}}<4.6 \mathrm{keV}$.

Another well-studied alternative dark matter model arises from ultra light axion (ULA) fields in the context of string axiverse [71, 88-91]. The mass of ULA particles lie in the range $m_{a} \simeq 10^{-33}-10^{-20} \mathrm{eV}$.

The ULA field obtains its initial condition after the spontaneous symmetry breaking in the early universe and behaves like a coherent scalar field. At an early time when the expansion rate $H \gg m_{a}$, the ULA behaves like a cosmological constant. At redshifts when $m_{a}<H$, the field rolls off and starts oscillating coherently around the nearest minima of the periodic potential. During this period, the average energy density of the field falls as $1 / a^{3}$ and therefore the background density acquires the characteristics of a CDM particle.

The adiabatic perturbations in the scalar field have a scale-dependent effective sound speed. At late times and sub-horizon scales, the effective sound speed is:

$$
c_{\mathrm{S}}^{2}=\frac{\frac{k^{2}}{4 m_{a}^{2} a^{2}}}{1+\frac{k^{2}}{4 m_{a}^{2} a^{2}}}
$$

At scales $k \gg m_{a} a$, the sound speed approaches the speed of light which prevents clustering at these scales. At late times, the sound speed approaches zero which causes the ULA density perturbation to behave as the cold dark matter perturbation.

The scale below which the matter perturbations are suppressed can be approximated as [71]:

$$
k_{m} \simeq\left(\frac{m}{10^{-33} \mathrm{eV}}\right)^{1 / 3}\left(\frac{100 \mathrm{kms}^{-1}}{c}\right) h \mathrm{Mpc}^{-1}
$$

A smaller $m_{a}$ yields a larger scale of matter suppression. The cosmologically relevant mass range of ULA is $10^{-25}-10^{-20} \mathrm{eV}$, which we consider here.

We compute the ULA matter power spectra using the code Axion $C A M B$ which is the modified version of the publicly-available code $\mathrm{CAMB}^{1}$.

In Fig 1, we show the small-scale matter power spectra for many WDM and ULA models which are of interest to us in this paper. To motivate our discussion in the later sections, we note that for the ULA models corresponding to: $m_{a}=$ $\left\{10^{-20}, 10^{-21}, 10^{-22}, 5 \times 10^{-23}, 10^{-23}\right\} \mathrm{eV}$, the matter power is $10 \%$ lower than the $\Lambda \mathrm{CDM}$ model for $k \simeq\{30,10,4,3,1.5\} \mathrm{h} \mathrm{Mpc}^{-1}$, respectively. The corresponding numbers for the WDM models are: $m_{\mathrm{wdm}}=\{0.3,1,1.5,2,3\} \mathrm{keV}$ and $k \simeq\{0.3,2,3,4,9\} \mathrm{h} \mathrm{Mpc}^{-1}$, respectively.

\footnotetext{
${ }^{1}$ available at https://github.com/dgrin1/axionCAMB
} 


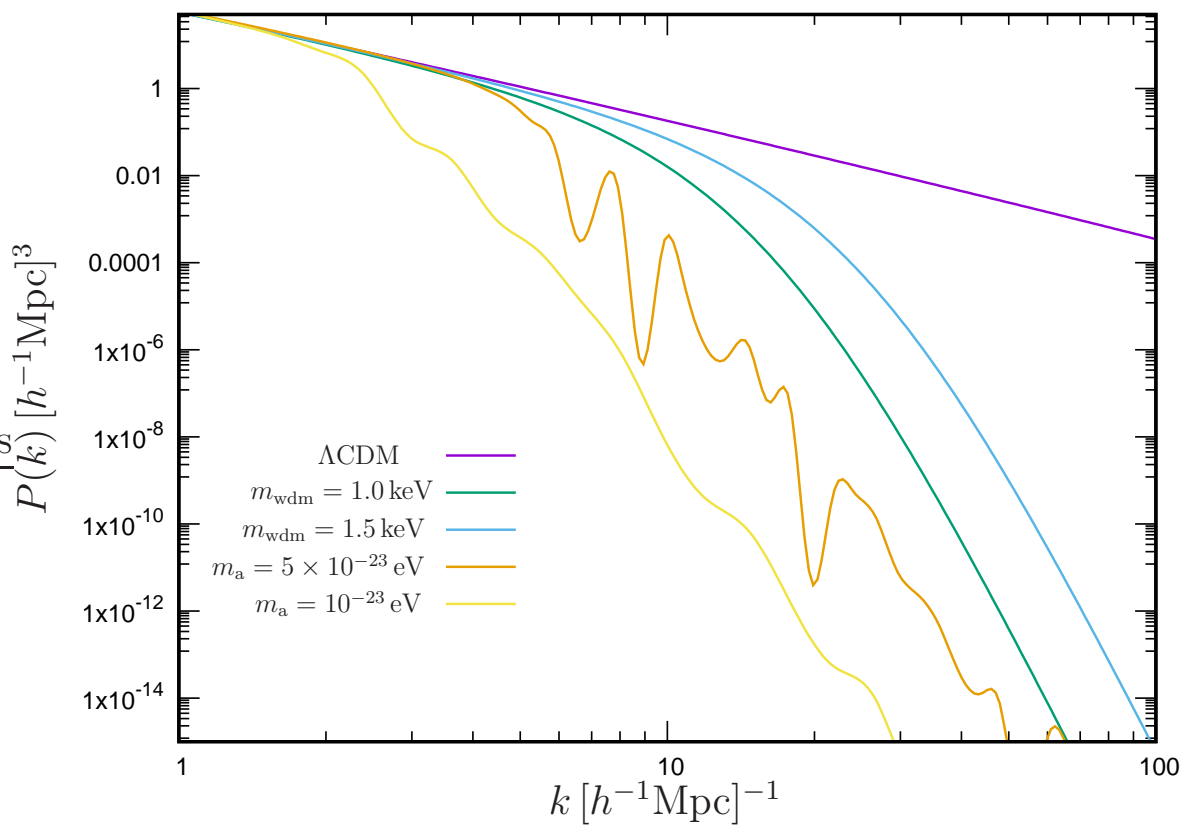

Figure 1. The figure displays the small-scale normalized matter power spectrum for the three models considered in this paper. The choice of parameters for the different models is listed in the inset.

\section{Simulating line-of-sight HI density fluctuations: Lyman- $\alpha$ clouds}

Hydrodynamical simulations show that the Lyman- $\alpha$ clouds are mildly non-linear regions in the IGM with density contrast $\delta \leq 10$ at high redshifts. This allows one to capture the ionization, thermal, and dynamical state of Lyman- $\alpha$ clouds using semianalytic models.

In this paper, we have followed the semi-analytic approach given in [47]. We briefly outline different steps in this process. The first step is the computation of the threedimensional baryonic matter power spectrum $P_{\mathrm{b}}^{(3)}(k, z)$ from the three-dimensional matter power spectrum $P_{\mathrm{m}}^{(3)}(k, z)$ :

$$
P_{\mathrm{b}}^{(3)}(k, z)=\frac{P_{\mathrm{m}}^{(3)}(k, z)}{\left(1+\lambda_{b}^{2} k^{2}\right)^{2}}
$$

Here $\lambda_{b} \equiv k_{J}^{-1}$ is the thermal Jeans scale:

$$
\lambda_{b}=\frac{1}{H_{0}}\left[\frac{2 \gamma k_{\mathrm{B}} T_{\mathrm{m}}(z)}{3 \mu m_{p} \Omega_{\mathrm{m}}}\right]^{1 / 2} \times(1+z)^{-1 / 2}
$$


All the parameters that appears in Eq. (3.2) have their usual meanings with $\mu=0.6$. For the parameters we use, $k_{J} \simeq 8.5 \mathrm{~h} \mathrm{Mpc}^{-1}$ at $z=3^{2}$

The relevant one-dimensional baryonic power spectra can be computed from the three-dimensional baryonic power spectrum (Eq. 3.1):

$$
\begin{array}{r}
P_{\mathrm{b}}^{(1)}\left(k_{1}, z\right)=\frac{1}{2 \pi} \int_{\left|k_{1}\right|}^{\infty} d k^{\prime} k^{\prime} P_{\mathrm{b}}^{(3)}\left(k^{\prime}, z\right) \\
P_{\mathrm{v}}^{(1)}\left(k_{1}, z\right)=\dot{a}^{2} k^{2} \frac{1}{2 \pi} \int_{\left|k_{1}\right|}^{\infty} \frac{d k^{\prime}}{k^{\prime 3}} P_{\mathrm{b}}^{(3)}\left(k^{\prime}, z\right) \\
P_{\mathrm{bv}}^{(1)}\left(k_{1}, z\right)=i \dot{a} k \frac{1}{2 \pi} \int_{\left|k_{1}\right|}^{\infty} \frac{d k^{\prime}}{k^{\prime}} P_{\mathrm{b}}^{(3)}\left(k^{\prime}, z\right)
\end{array}
$$

Here $k_{1}$ denotes the Fourier mode along the line of sight. $P_{\mathrm{b}}^{(1)}(k, z), P_{\mathrm{v}}^{(1)}(k, z)$ and $P_{\mathrm{bv}}^{(1)}(k, z)$ denote the line-of-sight density, velocity, and the cross power spectra of the density and the velocity fields, respectively.

Given the one-dimensional power spectra (Eq. (3.3), the correlated density and velocity fields are simulated by applying the Gram-Schimdt procedure on two independent Gaussian random fields (for details see e.g. [48] and references therein).

This process yields the correlated density and velocity fields in the Fourier space. we then apply inverse Fourier transform on these fields to obtain the corresponding real-space density and velocity fields, $\delta_{b}(x, z)$ and $v(x, z)$, respectively. The main aim of this paper is to study the redshift evolution of observables derived from Lyman- $\alpha$ data. Therefore, we simulate the density and the velocity fields for 40 different redshift bins each of width $\Delta z=0.1$ over the redshift range $2 \leq z \leq 6$. In each of these bins, there are $2^{14}$ points that resolve the Jeans scale $\lambda_{b}$ by at least a factor of 4 . The simulated fields are then smoothed to the instrumental resolution (discussed in detail later).

In the semi-analytic approach, the non-linearity of the density perturbations in the IGM is incorporated by assuming the density field to follow the lognormal distribution [47]. This allows us to relate the simulated baryonic density contrast, $\delta_{\mathrm{b}}(x, z)$ to the baryon number density $n_{\mathrm{b}}(x, z)$ as,

$$
n_{\mathrm{b}}(x, z)=A e^{\delta_{\mathrm{b}}(x, z)}
$$

$A$ is a normalization constant that can be determined by averaging the baryon number density:

$$
\left\langle n_{\mathrm{b}}(x, z)\right\rangle \equiv n_{0}(x, z)=A\left\langle e^{\delta_{\mathrm{b}}(x, z)}\right\rangle
$$

\footnotetext{
${ }^{2}$ The thermal Jeans' scale correspond to Jeans' mass $M_{J} \simeq 5 \times 10^{8} \mathrm{M}_{\odot}$ at $z \simeq 3$. The Jeans' mass in the unheated IGM at $z \simeq 20$ is $M_{J} \simeq 10^{5} \mathrm{M}_{\odot}$. After the re-heating and the reionization of the IGM in the redshift range $7<z<20$, the Jeans' mass increases and remains large in the post-reionization universe. The net impact of these processes is to prevent of growth of perturbations at sub-Jeans' scale for $z \lesssim 20$. While this would result in the suppression of the density contrast at these scales as compared to the larger scales, the suppression might not be as large as given in Eq. (3.1), which corresponds to a more conservative choice. This is partly justified as the reheating and reionization history of the universe during the era of cosmic dawn and the reionization is not yet well understood.
} 
Since the density perturbation $\delta_{\mathrm{b}}(x, z)$ is assumed to be Gaussian, we can write $\left\langle e^{\delta_{\mathrm{b}}(x, z)}\right\rangle$ as,

$$
\left\langle e^{\delta_{\mathrm{b}}(x, z)}\right\rangle=e^{\left\langle\delta_{\mathrm{b}}^{2}(x, z)\right\rangle / 2}
$$

Using Eqs. (3.5) and (3.6) in Eq. (3.4), the baryon number density $n_{\mathrm{b}}(x, z)$ can be expressed as:

$$
n_{\mathrm{b}}(x, z)=n_{0}(z) e^{\left(\delta_{\mathrm{b}}(x, z)-\left\langle\delta_{\mathrm{b}}^{2}(x, z)\right\rangle / 2\right)}
$$

where $n_{0}(z)$ gives the background number density of the baryons at a redshift $z$ :

$$
n_{0}(z)=\frac{\Omega_{\mathrm{b}} \rho_{c}}{\mu_{b} m_{p}}(1+z)^{3}
$$

Here $\mu_{b}=1.2$.

\section{Neutral hydrogen and the Lyman- $\alpha$ optical depth}

For comparison with Lyman- $\alpha$ data, we need the fluctuating component of the neutral hydrogen field along the line of sight, $n_{\mathrm{HI}}(x, z)$. The relation between the baryonic field (Eq. (3.5)) and the neutral hydrogen field can be computed by assuming ionization equilibrium in the IGM and the Lyman- $\alpha$ clouds, which are optically thin to the ionizing radiation. This gives us:

$$
n_{\mathrm{HI}}(x, z) \simeq \frac{\alpha[T(x, z)] n_{\mathrm{b}}(x, z)}{\Gamma_{c i}(x, z)+J(z) /\left[\mu_{e} n_{\mathrm{b}}(x, z)\right]}
$$

In writing Eq. (4.1), it has been implicitly assumed that the gas is highly ionized, as is expected for the physical parameters of the IGM. The temperature field, $T(x, z)=$ $T_{0}(z)\left[n_{\mathrm{B}}(x, z) / n_{0}(z)\right]^{\gamma-1}$, where $T_{0}$ gives the mean IGM temperature and $\gamma$ is the polytropic index of the gas. The variation of $\gamma$ provides information about the dynamical state of the Lyman- $\alpha$ clouds. $\alpha[T(x, z)]$ is the recombination coefficient and its value is determined by the temperature field at a given location. $\Gamma_{\mathrm{ci}}(x, z)$ is the coefficient of collisional ionization (in $\mathrm{cm}^{3} \mathrm{sec}^{-1}$ ) and $J(z)$ denotes the rate of photoionization (in $\mathrm{sec}^{-1}$ ). For a fully ionized gas, $\mu_{e}=1.07$ (see Eq. (25) in [92]). To model Lyman- $\alpha$ clouds, the free parameters have values in the ranges: $7000 \leq T_{0} \leq 15000 \mathrm{~K}$ and $1.3 \leq \gamma \leq 1.6[93]$

To compare the simulated data set against the observed flux decrement in the Lyman- $\alpha$ clouds, we need the optical depth $\tau(\nu)$ of the clouds for Lyman- $\alpha$ scattering as a function of frequency, which is given by:

$$
\tau(\nu)=\int n_{\mathrm{HI}}(x, z) \sigma_{a}\left(\frac{\nu}{a}\right) d l
$$

Here $n_{\mathrm{HI}}(x, z)$ gives the number density of the $\mathrm{HI}$ and $\nu$ is the observed frequency. The absorption cross section $\sigma_{a}$ is given by:

$$
\sigma_{a}=\frac{I_{a}}{b \sqrt{\pi}} V\left(\alpha, \frac{\nu-\nu_{a}}{b \nu_{a}}+\frac{v}{b}\right),
$$


where the parameter $b=\left(2 k_{\mathrm{B}} T / m_{p}\right)^{1 / 2}$ gives the velocity dispersion of the HI atoms, $v(x)$ is HI velocity field, $\alpha=2 \pi e^{2} \nu_{a} / 3 m_{e} c^{3} b=4.8548 \times 10^{-8} / b, I_{a}=4.45 \times 10^{-18}$ $\mathrm{cm}^{-2}$, and $V$ denotes the Voigt function.

For a temperature $T \simeq 10^{4} \mathrm{~K}$, these effects combine to yield us the optical depth $\tau$ as a function of redshift, which can be approximated as (e.g. [27]):

$$
\tau(z)=A\left(n_{\mathrm{B}} / n_{0}\right)^{2-0.7(\gamma-1)}
$$

where

$$
A=0.946\left(\frac{1+z}{4}\right)^{6}\left(\frac{\Omega_{b} h^{2}}{0.0125}\right)^{2}\left(\frac{T_{0}}{10^{4} \mathrm{~K}}\right)^{-0.7}\left(\frac{J}{10^{12} \mathrm{~S}^{-1}}\right)^{-1}\left(\frac{H(z)}{H_{0}}\right)^{-1}
$$

For the purpose of comparison with the data, we compute the Lyman- $\alpha$ effective optical depth $\tau_{\text {eff }}(z)$ as,

$$
\tau_{\text {eff }}(z)=-\log [\langle\exp (-\tau)\rangle],
$$

which is the observable that quantifies the decrease in the observed flux $\left(F \propto e^{-\tau}\right)$ as a function of redshift. The closed angular bracket $\langle.$.$\rangle in the above equation refers to$ the average of $\exp (-\tau)$ over all possible realizations of the optical depth $\tau \equiv \tau(z)$ for a given redshift bin.

\subsection{Data for $\tau_{\text {eff }}$}

The aim of this paper is to constrain certain dark matter models in which the small scale power ( $\leq$ a few Mpc) is suppressed in comparison with the usual $\Lambda$ CDM model. This motivates us to analyze high-resolution observations of the Lyman- $\alpha$ forest. We therefore consider the data compiled by [43]. This data measures of the Lyman- $\alpha$ optical depth $\tau_{\text {eff }}$ over a redshift range $2 \leq z \leq 4.2$ at an interval $\Delta z=0.2$ using a sample of 86 high-resolution, high S/N (Signal-to-Noise) quasar spectra. The data set consists of 16 (44) quasar spectra observed using HIRES (ESI) spectrographs with the Keck telescope, while the remaining 26 quasar spectra were obtained from with the MIKE instrument on Magellan. The HIRES and ESI spectrographs yield velocity resolutions $\Delta v \simeq 6-8 \mathrm{~km} / \mathrm{s}$ and $\Delta v \simeq 33-44 \mathrm{~km} / \mathrm{s}$, respectively, and the MIKE spectrograph operates at $\Delta v \simeq 11-14 \mathrm{~km} / \mathrm{s}$. Most of the quasar spectra obtained using HIRES and ESI spectrographs are used to study the Damped Lyman Alpha (DLA) systems $[94,95]$ and have $\mathrm{S} / \mathrm{N} \geq 15$ pixel $^{-1}$, whereas the spectra obtained using MIKE spectrograph were analyzed for the Super Lyman Limit (SLL) systems given in [96] and has $\mathrm{S} / \mathrm{N} \geq 10$ pixel $^{-1}$.

For an expanding universe, the velocity resolution width $\Delta v$ corresponds to a line-of-sight comoving length: $\Delta r=\Delta v(1+z) / H(z)$. This gives $\Delta r \simeq 0.01 \mathrm{Mpc}$ for $\Delta v=1 \mathrm{~km} / \mathrm{s}$ at $z=3$. This allows us to express the observed velocity resolution in terms of the largest line-of-sight Fourier mode that the data can probe (Eq. (3.3)). One can readily show that the lowest resolution of the data we use $(\Delta v \simeq 40 \mathrm{~km} / \mathrm{s})$ is comparable to the Jeans' scale (Eq. 3.2). In our theoretical model, the baryonic power spectrum is sharply cut off at the Jeans' scale (Eq. (3.1)), which means that even though the data resolves the Jeans' scale we do not expect the data to contain 
information on scales $k>k_{J}$. In our analysis, we first obtain the optical depth for individual clouds (Eq. (4.2)), which is followed by the computation of the transmitted flux $\propto \exp \left(-\tau_{i}(\nu)\right)$ as a function of frequency. The simulated transmitted fluxes are then smoothed with a velocity resolution $\Delta v=7.5 \mathrm{~km} / \mathrm{s}$ for comparison with data. We note that our results are insensitive to the choice of the velocity resolution if the length scale corresponding to the velocity resolution is smaller or comparable to the thermal Jeans' scale (Eq. 3.2), which is the smallest scale we can probe in our study.

More Recently, [45] have estimated $\tau_{\text {eff }}$ over a larger redshift range $2 \leq z \leq 5$ using a sample of 6065 moderate-resolution quasar spectra drawn from SDSS DR7 [97]. However, the velocity resolutions of the quasar spectra are in the range $\Delta v \simeq$ $143-167 \mathrm{~km} / \mathrm{s}$, which correspond to length scales considerably larger than the Jeans' scale and therefore are not suitable for our analysis ${ }^{3}$

\section{$5 \quad$ Results}

Our analysis is based on comparing the measured effective optical depth against the simulated optical depth as a function of redshift. We compute the effective optical depth $\tau_{\text {eff }}(z)$ from the simulated line-of-sight HI field for three different cosmological models - $\Lambda$ CDM, WDM and ULA models. The dark matter model is constrained from comparing the slope of the redshift dependence of the effective optical depth for different models.

We simulate the Lyman- $\alpha$ effective optical depth $\tau_{\text {eff }}(z)$ over the redshift range $2 \leq z \leq 6$ at a redshift interval of $\Delta z=0.1$ using the method detailed in the last section. Throughout this section, the following parameters, unless otherwise specified, are used for computing $\tau_{\text {eff }}(z): J=1.5 \times 10^{-12} \mathrm{~s}^{-1}, T_{0}=1.3 \times 10^{4} \mathrm{~K}, \gamma=1.4$, and $\Delta v=7.5 \mathrm{~km} / \mathrm{s}$.

The Lyman- $\alpha$ effective optical depth $\tau_{\text {eff }}(z)$ at a given redshift $z$ can be calculated by replacing the ensemble average defined in Eq. (4.6) by an average over optical depths of individual clouds in the simulated HI field: $\tau_{\text {eff }}(z)=-\log \left[\sum_{i} \exp \left(-\tau_{i}\right) / N\right]$ where $N$ and $\tau_{i}$ denote the number of the Lyman- $\alpha$ clouds and the optical depth of the $i$-th Lyman- $\alpha$ cloud at that redshift, respectively. The HI column density of a Lyman- $\alpha$ cloud at a given redshift depends on both the baryonic density perturbation $\delta$ and the background baryon density at that redshift. In a matter-dominated universe, a reasonable assumption for the standard $\Lambda \mathrm{CDM}$ model at $z>2$, the matter density contrast, $\delta \propto(1+z)^{-1}$ while the background density $n_{\mathrm{HI}} \propto(1+z)^{3}$. This gives: $\tau_{i} \propto n_{\mathrm{HI}}^{2} H^{-1}(z)(1+\delta)^{\beta}$ where $\beta=2-0.7(\gamma-1)$. Using $\delta \gg 1$, we get, $\tau_{i} \propto(1+z)^{4.5-\beta}$. Here $H(z) \propto \Omega_{m}^{0.5}(1+z)^{1.5}$ is the Hubble parameter. The optical depth of an individual Lyman- $\alpha$ cloud, $\tau_{i}$, evolves sharply with the redshift. A part of this change arises from the evolution of background quantities (Hubble's parameter and background density)

\footnotetext{
${ }^{3}$ We note the mean flux decrement is independent of spectral resolution and therefore in principle our proposed method should work with low-resolution data sets also. However, continuum subtraction does depend on spectral resolution and it impacts the mean flux decrement. Hence, we prefer to work with the highest spectral resolution data set.
} 
but an additional change comes from the density contrast and equation of state $\gamma$ of the clouds.

At any given redshift, the range of optical depths of clouds is governed by the log-normal density distribution (Eq. (3.4)). To understand the redshift evolution of this distribution, we note that the line-center cross-section for Lyman- $\alpha$ scattering for a non-expanding region of temperature $T \simeq 10^{4} \mathrm{~K}$ is $\simeq 5 \times 10^{-14} \mathrm{~cm}^{2}$. As the column densities of most clouds lie in the range $10^{13}-10^{15} \mathrm{~cm}^{2}$, many clouds are optically thick at any redshift and a larger fraction of clouds is optically thick at higher redshifts.

To further analyze the implication of this dynamics, we compare the redshift evolution of $\tau_{\text {avg }}(z) \equiv \sum_{i} \tau_{i} / N$ with the observationally determined effective optical depth, $\tau_{\text {eff }}(z)$. If optical depths of all the Lyman- $\alpha$ clouds are small, $\tau_{i} \ll 1$, then $\tau_{\text {avg }} \simeq \tau_{\text {eff }}$ at all redshifts. In the other limit, if all the clouds are optically thick $\tau_{i}>1$, the redshift evolution of $\tau_{\text {avg }}$ is the same as in the first case, but the $\tau_{\text {eff }}$ doesn't change with the redshift. In the intermediate situation which is suitable for the allowed cosmological models, a fraction of clouds are optically thick at any redshift and this fraction increases at larger redshifts. This implies that the redshift evolution of $\tau_{\text {eff }}$ is always flatter than the redshift dependence of $\tau_{\text {avg }}$.

In figure 2, we show the redshift dependence of Lyman- $\alpha$ effective optical depth $\tau_{\text {eff }}(z)$ and the average Lyman- $\alpha$ optical depth $\tau_{\text {avg }}(z)$ for the $\Lambda$ CDM model. We notice that $\tau_{\text {eff }}(z) \simeq \tau_{\text {avg }}(z)$ at $z \simeq 2$ but $\tau_{\text {ave }}(z)$ diverges from $\tau_{\text {eff }}(z)$ at high redshifts. As anticipated, the slope of $\tau_{\text {eff }}(z)$ is smaller than that of $\tau_{\text {avg }}(z)$.

\subsection{Effective optical depth and dark matter models}

The main aim of this paper is to constrain dark matter models using the redshift evolution of $\tau_{\text {eff }}$. The alternative dark matter models we consider are described in detail in section 2. The discussion in the foregoing provides motivation of this aim and we elaborate it further here.

Both the alternative models we consider yield a suppression of matter power at small scales as compared to the usual $\Lambda$ CDM model (Figure 1). The scales at which the power is suppressed is determined by the mass of particles in both cases. As discussed above, the optical depth of an individual cloud depends on both the background density and the density contrast, $\delta$. While the background density is left unchanged in alternative dark matter models we consider, the density contrast decreases owing to the decrement in matter power at small scales.

We next study the evolution of $\tau_{\text {eff }}(z)$ and $\tau_{\text {avg }}(z)$ in these models relative to the $\Lambda$ CDM model, which is shown in Figure 2. In Figure 3 the relevant plots are displayed. As expected $\tau_{\text {avg }}(z)$ is smaller in alternative dark matter models because of the decrement of density contrast in these models. The difference between the average optical depth $\tau_{\text {avg }}(z)$ for these models and that for the $\Lambda$ CDM model is seen to be negative in the redshift range $2 \leq z \leq 6$.

This also allows us to understand the difference of the average optical depth between the two alternative dark matter models. The scales at which the suppression of the matter power occurs in the WDM model for $m_{\mathrm{wdm}}=0.3 \mathrm{keV}$ are larger as 


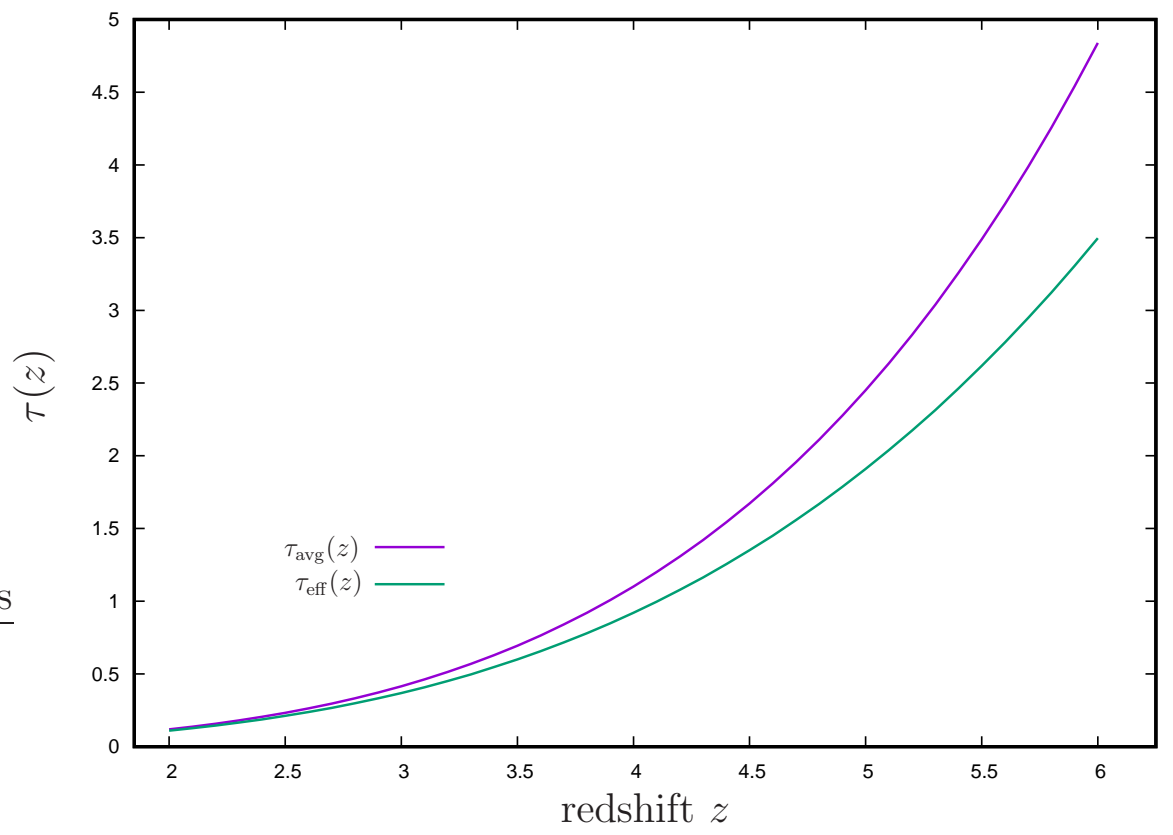

Figure 2. The figure shows the redshift evolution of the effective and the average optical depths, $\tau_{\text {eff }}(z)$ and $\tau_{\text {avg }}(z)$, for the $\Lambda$ CDM model for the following parameters: $J=1.5 \times$ $10^{-12} \mathrm{~s}^{-1}, T_{0}=1.3 \times 10^{4} \mathrm{~K}, \gamma=1.4$ and $\Delta v=7.5 \mathrm{~km} / \mathrm{s}$.

compared to the ULA model for $m_{\mathrm{a}}=10^{-23} \mathrm{eV}$. The matter power gets suppressed roughly by a factor $\simeq 10$ in the range $k \simeq 0.3-0.5 \mathrm{~h} \mathrm{Mpc}^{-1}$ for $m_{\mathrm{wdm}}=0.3 \mathrm{keV}$, whereas, for $m_{\mathrm{a}}=10^{-23} \mathrm{eV}$, the matter power decreases by a factor $\simeq 3$ for the range $k \simeq 0.3-1.0 h \mathrm{Mpc}^{-1}$. This explains the smaller values of $\tau_{\mathrm{avg}}(z)$ for the WDM model for $m_{\mathrm{wdm}}=0.3 \mathrm{keV}$ as compared to the ULA models for $m_{\mathrm{a}}=10^{-23} \mathrm{eV}$ over the entire redshift range.

Figure 3 also shows the effective optical depth for different models. However, unlike the monotonic behavior of average optical depth, we notice a transition at $z \simeq 3^{4}$. The discussion in the foregoing allows us to understand this transition. At small redshifts, the $\Lambda$ CDM model has higher effective optical depth for the following reasons: (a) the optical depth for an individual cloud, $\tau_{i}<1$, for most clouds for all the models and (b) the optical depth of individual clouds $\tau_{i}$ is higher for the $\Lambda$ CDM model owing to a larger density contrast. So the effective optical depth converges to the average optical depth at small redshifts (Figure (2)), which is higher for the $\Lambda$ CDM models as compared to the alternative dark matter models. This also means that at higher redshifts a larger number of clouds become optically thick for the $\Lambda$ CDM which causes the effective optical depth to partially saturate and consequently the redshift evolution of the effective optical depth has a smaller slope. On the other hand, for the

\footnotetext{
${ }^{4}$ The redshift at which the transition occurs depends on the choice of dark matter model parameters. For instance, the transition redshift shifts to smaller redshifts as $m_{\mathrm{wdm}}$ is increased.
} 
alternative dark matter models, the effective optical depth tracks the average optical depth up to a much higher redshifts and therefore has a sharper slope in the redshift space. This explains the transition from a positive difference to a negative difference in the effective optical depth between the $\Lambda \mathrm{CDM}$ and the alternative dark matter models. This also explains the evolution of the difference between the two alternative dark matter models.

To illustrate this aspect further, we show the relative difference of the average and effective optical depths between the $\Lambda$ CDM model and the alternative dark matter models in Figure 4. We define the relative difference $\Delta \tau(z)$ as, $\Delta \tau(z)=$ $\left[\tau^{\text {model }}(z) / \tau^{\mathrm{CDM}}(z)-1\right] \times 100 \%$ for a given model where $\tau^{\mathrm{CDM}}$ and $\tau^{\text {model }}$ refer to corresponding quantities for the $\Lambda \mathrm{CDM}$ model and one of the alternative dark matter models considered in our analysis. As expected, the relative differences $\Delta \tau_{\text {avg }}(z)$ are negative throughout the redshift range $2 \leq z \leq 6$ (see the left panel of Figure 4). For the ULA model with $m_{\mathrm{a}}=10^{-23} \mathrm{eV}$, the relative difference varies in the range $\Delta \tau_{\text {avg }}(z) \simeq 3-6 \%$ over the redshift range $2 \leq z \leq 6$. For the WDM model with $m_{\mathrm{wdm}}=0.3 \mathrm{keV}$, the difference increase by factors $\sim 1.3-1.6$ over the redshift range.

We further see that the relative differences $\Delta \tau_{\text {eff }}$ are negative at redshifts $z<3$ and positive at redshifts $z>3$ (see the right panel of Figure 4). For $m_{\mathrm{a}}=10^{-23} \mathrm{eV}$, the relative difference varies from $\Delta \tau_{\text {eff }}(z) \sim-4 \%$ to $\Delta \tau_{\text {eff }}(z) \sim 12 \%$ over the redshift range $2 \leq z \leq 6$. The WDM models show similar cross-over from negative to positive difference as is seen in the Figure 4 (right panel). We note that for the WDM model with $m_{\mathrm{wdm}}=0.3 \mathrm{keV}$, the difference increases by a factor $\sim 1.5$ over the redshift range.

\subsection{Comparison with data}

In Figure 5 we display the redshift evolution of $\tau_{\text {eff }}(z)$ for a few dark matter models along with the high-resolution Lyman- $\alpha$ data ([43]).

For a detailed statistical analysis, we carry out a likelihood analysis that compares the simulated effective optical depths with the data.

In addition to the masses: $m_{\mathrm{wdm}}$ and $m_{\mathrm{a}}$, we consider the following four parameters related to the ionization, thermal and dynamical state of Lyman- $\alpha$ clouds in our analysis: $J(z), \alpha, T_{0}$ and $\gamma$.

The ionization and thermal history in the redshift range $2 \lesssim z \lesssim 5$ is known from the observation of Lyman- $\alpha$ clouds (for a comprehensive review see e.g. [98] and references therein). The temperature of the IGM $T_{0} \simeq 10^{4} \mathrm{~K}$ with no discernible redshift evolution for $z>3.5$. The reionization of singly-ionized helium raises the temperature by approximately a factor of 1.5 for $z<3.5$ [99]. This is consistent with the fact that the temperature of optically-thin photoionized hydrogen gas is nearly independent of the magnitude of the ionizing flux with a weak dependence on the spectral index $[98,100]$. We therefore expect $T_{0}$ to be nearly independent at higher redshifts if the gas is photoionized and the spectral index of the ionizing sources doesn't change appreciably. The equation of state parameter $\gamma$ has been extensively studied using hydrodynamical simulations and its acceptable range has been determined by comparison with Lyman- $\alpha$ forest data [93, 101]. The Lyman- $\alpha$ forest data suggests that the ionizing intensity $J(z)$ doesn't evolve in the redshift range $2<z<4.2$ [102]. 
However, its redshift evolution at higher redshifts remains uncertain. We account for the redshift evolution of the ionizing intensity $J(z)$ by assuming: $J(z)=J_{0}(1+z)^{\alpha}$; here $J_{0}$ and $\alpha$ are constants.

Based on these considerations, we vary $J_{0}, \alpha, T_{0}$ and $\gamma$ over the following ranges (with a flat prior) in the likelihood analysis: $0.7 \times 10^{-12} \leq J_{0} \leq 4 \times 10^{-12},-1.2 \leq \alpha \leq 1$, $0.7 \times 10^{4} \leq T_{0} \leq 2.3 \times 10^{4}$ and $1.4 \leq \gamma \leq 2.2$. For the WDM and ULA models, the masses $m_{\mathrm{wdm}}$ and $m_{\mathrm{a}}$ are varied over the ranges: $0.1 \mathrm{keV} \leq m_{\mathrm{wdm}} \leq 4.6 \mathrm{keV}$ and $10^{-24} \mathrm{eV} \leq m_{\mathrm{a}} \leq 5 \times 10^{-22} \mathrm{eV}$. Before embarking on the details of likelihood analysis, we discern the structure of degeneracy of the proposed five parameter likelihood function. Eqs. (4.4)-(4.6) show the dependence of effective optical depth on all the parameters. The alternative dark matter impacts the effective optical depth through the baryon density $n_{b} \equiv n_{0}\left(1+\delta_{B}\right)$ in Eq. (4.4). For mildly non-linear regions, $\delta_{B} \simeq 10$, $n_{b} \simeq n_{0} \delta_{B}$. For alternative dark matter models, $\delta_{B}$ is obtained by simulating the density and velocity fields from a different matter power spectrum. So if a combination of the other modelling parameters/functions, needed to infer the density field from the observed flux decrement $\left(\gamma, T_{0}\right.$, and $\left.J(z)\right)$, can entirely compensate for the change in $\delta_{B}$ over the redshift range of interest, the proposed impact of the change in the cosmological model would be unobservable. Eqs. (4.4)-(4.6) also allow us to compare the efficacy of our approach with the more direct method, which is normally based on the simulation of the three-dimensional density field using hydrodynamical simulations. This allows one to draw the density field along many line-of-sights. Eqs. (4.4)-(4.6) yield the line-of-sight transmitted flux field as a function of frequency, which is then used to compute transmitted flux correlation function. The correlation function from the simulated data can then be compared to the Lyman- $\alpha$ data. As Eqs. (4.4)-(4.6) are needed to compare the theoretical predictions with the data for this method also, it also partly shares the degeneracy structure of our proposed method.

However, while our method is based on using just one quantity, average overdensity, at a given redshift, the other method computes flux correlation function at any redshift and therefore is also sensitive to the spatial variation of the density field at any given redshift, while it can also use information from different redshifts. This method, therefore, retains more information than our approach. And, given the uncertainty in modelling parameters, it is expected to yield better constraints on the dark matter masses.

It is difficult to quantify the nature of the degeneracy analytically as the average overdensity is computed from a simulation and $\tau_{\text {eff }}$ is a highly non-linear function of modelling parameters. Eq. (4.4) only partially captures the nature of this effect as the Lyman- $\alpha$ clouds that most impact the redshift evolution of $\tau_{\text {eff }}$ are the ones with $\tau \simeq 1$ (Figure 4). However, Eq. (4.4) strongly suggests that the only models that can be constrained using our method are the ones for which the change in overdensity is larger than obtained by changing the modelling parameters within the prior range. This also means that we expect the bound on dark matter mass to weaken as the prior range is expanded. To study this further, we explored the dependence of the dark matter posterior probability on the modelling parameters by expanding the range of priors for each parameter. We found that the dependence on the prior is negligible if 
the maximum of the posterior probability of the relevant parameter lies in the prior range. For $\gamma$ the maximum of the posterior probability is around 1.1 which is outside the original range $(1.4 \leq \gamma \leq 2.2)$. We extended the prior range to encompass the maximum $(0.5 \leq \gamma \leq 2.2)$ and find a discernible but small weakening of the bound on dark matter masses. This also means that a better understanding of priors from complementary data might allow better determination of dark matter masses using our method.

Our analysis yields likelihood function as a function of the parameters, $J_{0}, \alpha, T_{0}$, $\gamma$, and either of the two masses, $m_{\mathrm{wdm}}$ or $m_{\mathrm{a}}$. As our method of constraining the alternative dark matter models relies upon the redshift evolution of $\tau_{\text {eff }}$, we first investigate the possible degeneracy between the masses of alternative dark matter particles and $\alpha$, which gives the redshift evolution of the ionizing radiation. We marginalize over the parameters $J_{0}, T_{0}, \gamma$ to obtain the marginalized joint likelihood of $\alpha$ and the masses: $m_{\mathrm{wdm}}$ and $m_{\mathrm{a}}$, for the WDM and the ULA models respectively. We find that the parameters: $\alpha$ and the masses: $m_{\mathrm{wdm}}$ and $m_{\mathrm{a}}$ are weakly anti-correlated. We marginalize the joint likelihood over the masses: $m_{\mathrm{wdm}}$ and $m_{\mathrm{a}}$ to get the marginalized posterior likelihood of the parameter: $\alpha$ for the WDM and the ULA models, respectively. The posterior probability of $\alpha$ is displayed in Figure 6 . We notice that the marginalized posterior likelihood of the parameter $\alpha$ peaks at $\alpha=-0.1$ (left panel) and $\alpha=-0.05$ (right panel), respectively, for the WDM and the ULA models. The peak values of the parameter $\alpha$ for both the WDM and the ULA models are consistent with $\alpha=0$ i.e. a redshift-independent background hydrogen-ionizing intensity, in agreement with the findings of [102]. In Figure 8 we show contour plots in the spectral index $\alpha$ and dark matter masses plane, which allows a further assessment of the dependence of dark matter mass on modelling parameters. In particular, the contour plots capture the degeneracy between dark matter masses and the evolution of ionizing radiation, which is difficult to determine from theoretical considerations

To get the posterior likelihoods of the masses for the WDM and the ULA models, we further marginalize the joint likelihood of $\alpha$ and the masses over the parameter $\alpha$. In Figure 7 , we show the posterior probabilities of the WDM mass $m_{\text {wdm }}$ (left panel) and the ULA mass $m_{\mathrm{a}}$ (right panel). The posterior probabilities peak at $m_{\mathrm{wdm}} \simeq$ a few $\mathrm{keV}$ for the WDM model and at $m_{\mathrm{a}} \simeq 5 \times 10^{-22} \mathrm{eV}$ for the ULA model and are flat for larger masses. As the $\Lambda \mathrm{CDM}$ model is a limit of these models for large masses, the data are seen to be consistent with the $\Lambda$ CDM model. The posterior probabilities fall for smaller masses which shows that the data are sensitive to the change in the matter power at small scales. Figure 7 shows that $1-\sigma$ (defined as the value at which the posterior probability is nearly 0.68 ) bounds from our analysis are: $m_{\mathrm{wdm}} \gtrsim 0.7 \mathrm{keV}$ and $m_{a} \gtrsim 2 \times 10^{-23} \mathrm{eV}$.

The discussion in section 2 allows us to understand these results. For $m_{\mathrm{wdm}} \simeq$ $3 \mathrm{keV}$, the matter power is smaller than the $\Lambda$ CDM model by around $10 \%$ at $k \simeq$ $9 \mathrm{hMpc}^{-1}$. The corresponding scale for $m_{a}=2 \times 10^{-22} \mathrm{eV}$ is $k \simeq 4 \mathrm{hMpc}^{-1}$. For the $1 \sigma$ bounds on the dark matter masses, the matter power is smaller than that for the $\Lambda \mathrm{CDM}$ model by approximately $10 \%$ at scales $k \simeq 2 \mathrm{hMpc}^{-1}$. This suggests that our proposed probe is sensitive to scales comparable to or larger than the Jeans' scale by 
a factor of 2. The posterior probability doesn't fall sharply for smaller masses which suggests that a range of even larger scales impact the observable. Figure 4 shows that the redshift coverage of the data is partly responsible for this behavior, as the deviation of the models corresponding to the peak of the posterior probability is less than $3 \%$ at $z \simeq 4.2$, which is the largest redshift in the data. This difference triples for $z \simeq 6$ which means the higher redshift data is a better discriminator between the different models. In the following subsection, we shall compare our theoretical predictions with a simulated data set that spans a redshift range: $2 \leq z \leq 6.5$.

The Lyman- $\alpha$ data can be analyzed in many different ways to probe the matter power spectrum at small scales. One way is to model the the one-dimensional power spectrum of the observed flux field along the line of sight using three-dimensional power spectra at any given redshift (Eq. (3.3)). Such modelling requires high-resolution hydrodynamical simulations. This method has been successfully applied to probe the matter power spectrum for over two decades now (e.g. [62, 63, 77-79, 103, 104]). Both the WDM and ULA models have been constrained using this approach $([62,63,76])$. The resultant upper bounds on the masses are: $m_{\text {wdm }} \gtrsim 3 \mathrm{keV}$ and $m_{a} \gtrsim 10^{-21} \mathrm{eV}$, which are significantly stronger than our results. These bounds suggest that such probes are sensitive to $k \simeq 10 \mathrm{hMpc}^{-1}$, while, as discussed above, our analysis can only probe scales larger than $k \simeq 2 \mathrm{hMpc}^{-1}$ (see the discussion in section 2). As this is partly owing to the redshift coverage of the high-resolution Lyman- $\alpha$ data, we next assess the efficacy of our proposed method using simulated data.

\subsection{Comparison with simulated data}

We simulate synthetic data sets for the $\Lambda$ CDM model in the redshift range $2<z<6.5$ and at redshift intervals $\Delta z=0.1$. The following parameters are used for computing $\tau_{\text {eff }}: J=1.5 \times 10^{-12} \mathrm{~s}^{-1}, T_{0}=1.3 \times 10^{4} \mathrm{~K}, \gamma=1.4$, and $\Delta v=7.5 \mathrm{~km} / \mathrm{s}$. The error at each data point is obtained by drawing from a Gaussian random variable of standard deviation $\sigma=p \tau_{\text {eff }}$. Here $p$ is chosen in the range 0.1 and $0.05^{5}$

We use the same priors on the parameters: $J, T_{0}$ and $\gamma$, as in the previous section. The parameters $J_{0}, \alpha, T_{0}$ and $\gamma$ are marginalized to obtain the posterior probability of masses $m_{\mathrm{wdm}}$ and $m_{\mathrm{a}}$ for the WDM and the ULA models respectively. In Figure 9, we show the posterior probabilities of the masses for $p=0.05$. For comparison, the posterior probabilities from Figure 7 are also shown.

From Figure 9, the 1- $\sigma$ forecasts on dark matter masses are: $m_{\mathrm{wdm}} \gtrsim 1.5 \mathrm{keV}$ and $m_{a} \gtrsim 7 \times 10^{-23} \mathrm{eV}$. This is significant improvement over the constraints obtained from the data set of [43]. This result satisfies our theoretical expectation (Figure 4) that the higher redshift data should provide better constraints. We also assess the impact of the error bar (given by the choice of $p$ ) in the simulated data points on the posterior probability. For $p=0.1$, we still find substantial improvement but the constraints on WDM mass are found to less sensitive to the choice of $p$.

While the comparison of the simulated data sets with the theory bears out the efficacy of our proposed method, the forecast bounds are still weaker than those obtained

\footnotetext{
${ }^{5}$ For comparison, $p$ varies in the following range for the existing data sets: $p=0.15-0.03([43])$ and $p=0.1-0.02([45])$.
} 
from hydrodynamical simulations. As already noted (see footnote 2), our prescription of implementing the physics close to the Jeans' scale results in a sharp cut off in the matter power for scales corresponding to $k_{J} \simeq 5 \mathrm{Mpc}^{-1}$, which is probably a factor of a few larger than the scales accessible to hydrodynamical simulations.
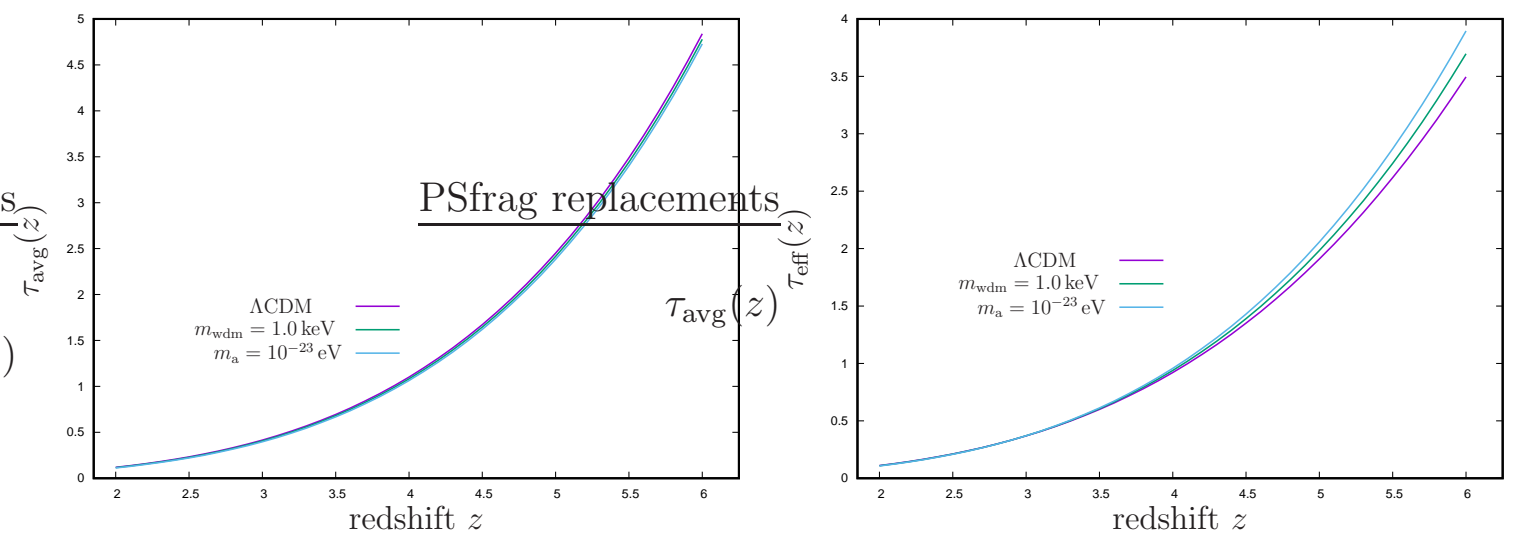

Figure 3. The figure displays the redshift evolution of the average and effective optical depths, $\tau_{\text {avg }}(z)$ and $\tau_{\text {eff }}(z)$, for $\Lambda$ CDM, WDM, and ULA models. The other modelling parameters are the same as in Figure 2.
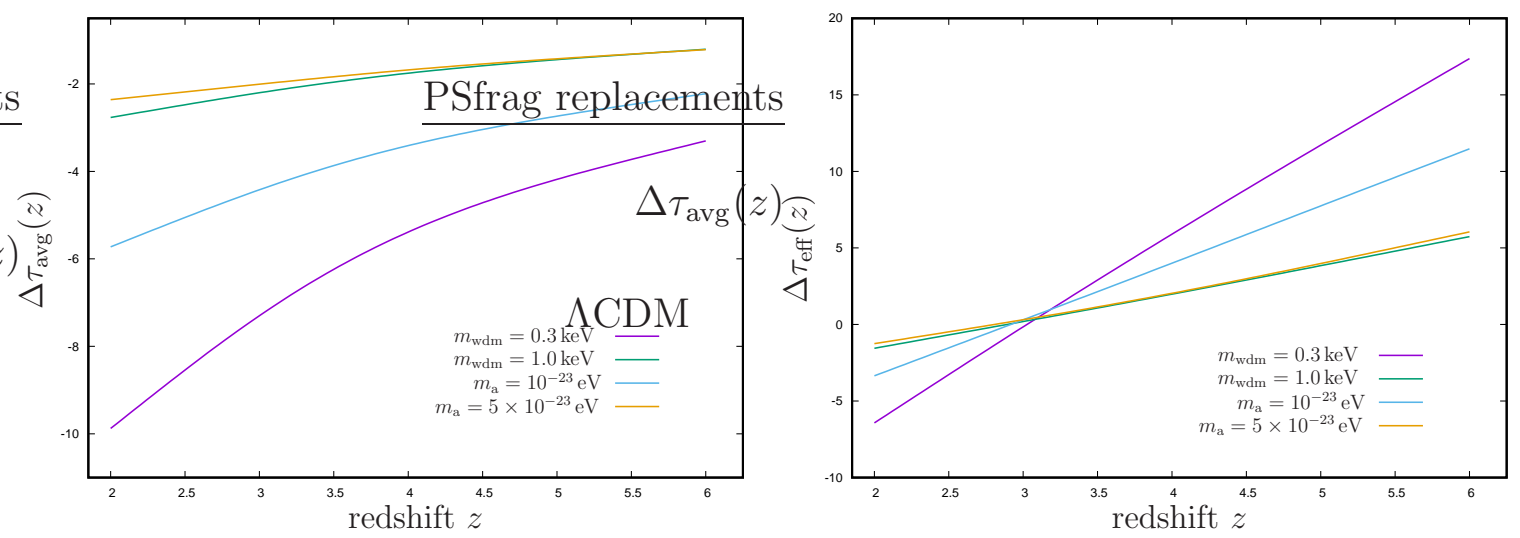

Figure 4. The redshift evolution of the relative differences $\Delta \tau_{\mathrm{avg}}(z)$ and $\Delta \tau_{\text {eff }}(z)$, comparing the $\Lambda$ CDM model with the WDM and ULA models, are plotted.

\section{Summary and Conclusions}

It is well known that the Lyman- $\alpha$ forest in the redshift range $2<z<5$ are an excellent probe of the matter power spectrum for scales as small as the Jeans' scale of the IGM, $k_{J} \simeq 5-7 \mathrm{Mpc}^{-1}$. 


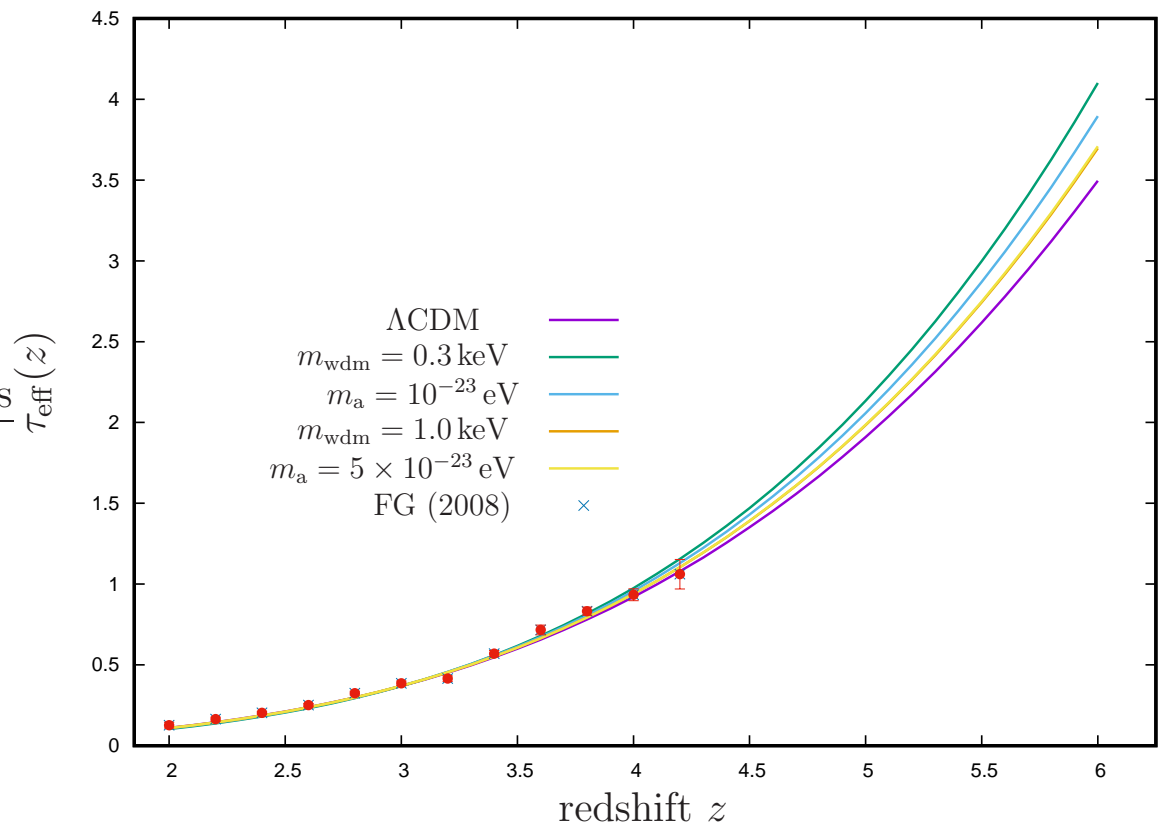

Figure 5. The figure compares the simulated $\tau_{\text {eff }}(z)$, for one set of parameters, for the $\Lambda \mathrm{CDM}$, the WDM, and the ULA models, with the data [43]. The other modelling parameters are the same as in Figure 2.
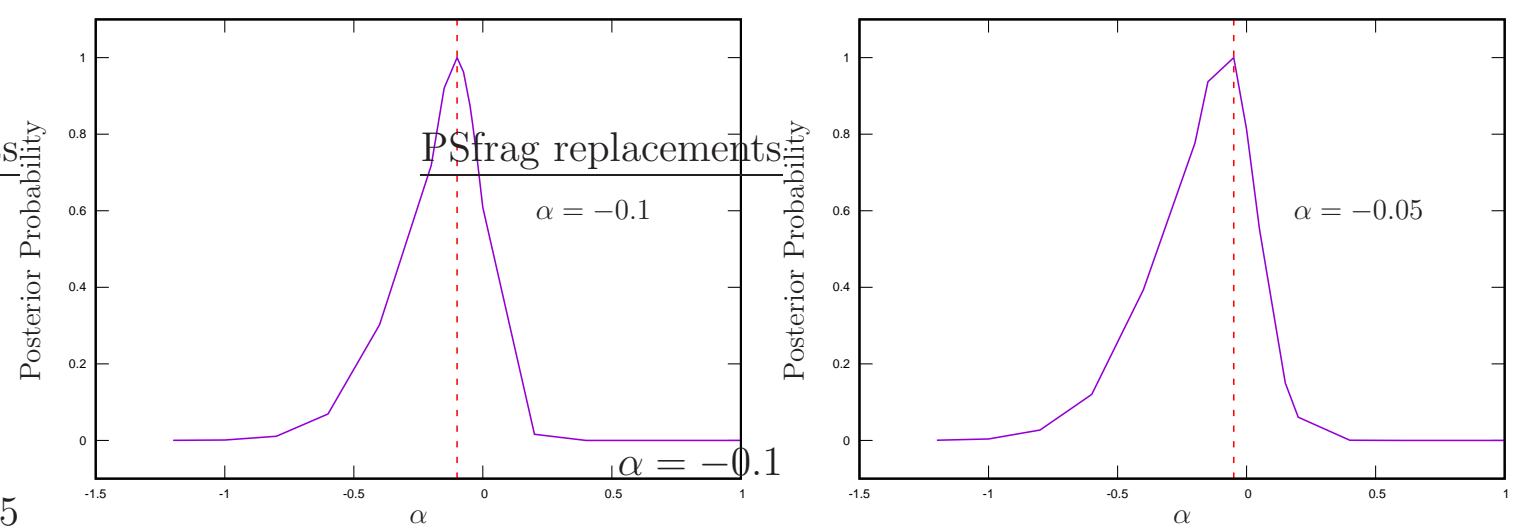

Figure 6. The figure shows the posterior probabilities of $\alpha$ (see text for details) for the WDM (left panel) and the ULA (right panel) models.

In this work, we use semi-analytic modelling of Lyman- $\alpha$ clouds and the redshift evolution of the effective optical depth to distinguish between dark matter models whose matter power spectra differ from the $\Lambda$ CDM model at small scales. This approach has been shown to be sensitive to the small scale matter power $([48])$. We 

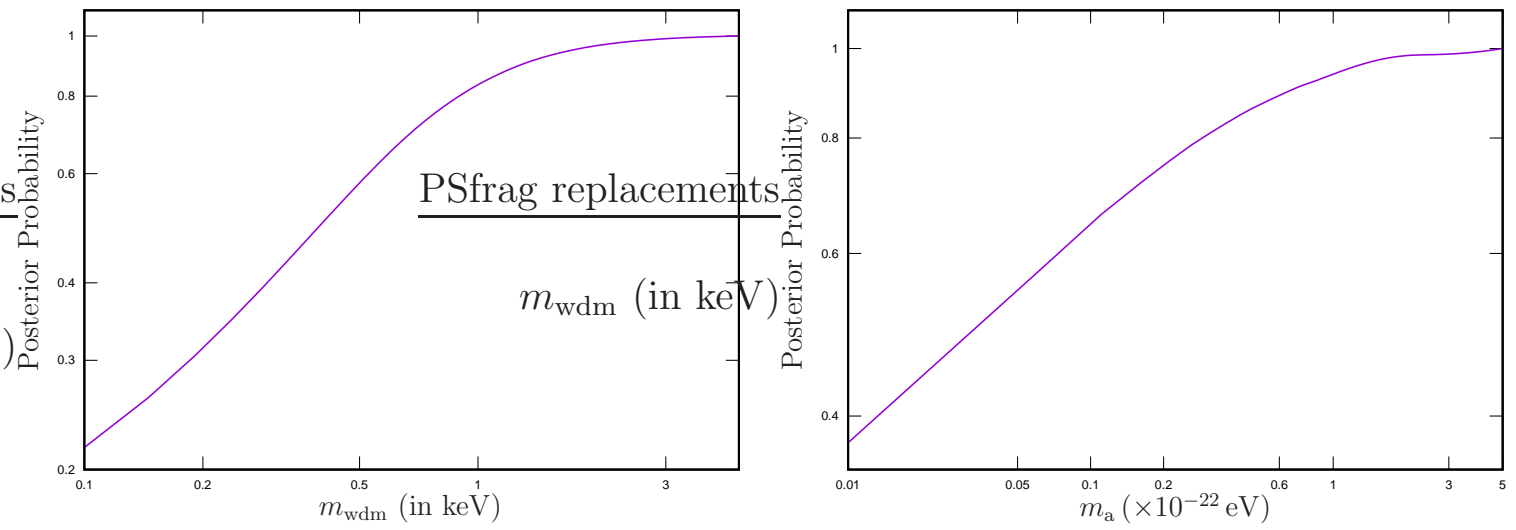

Figure 7. The posterior probabilities of $m_{\mathrm{wdm}}$ and $m_{\mathrm{a}}$ for the WDM (left panel) and the ULA (right panel) models are displayed.
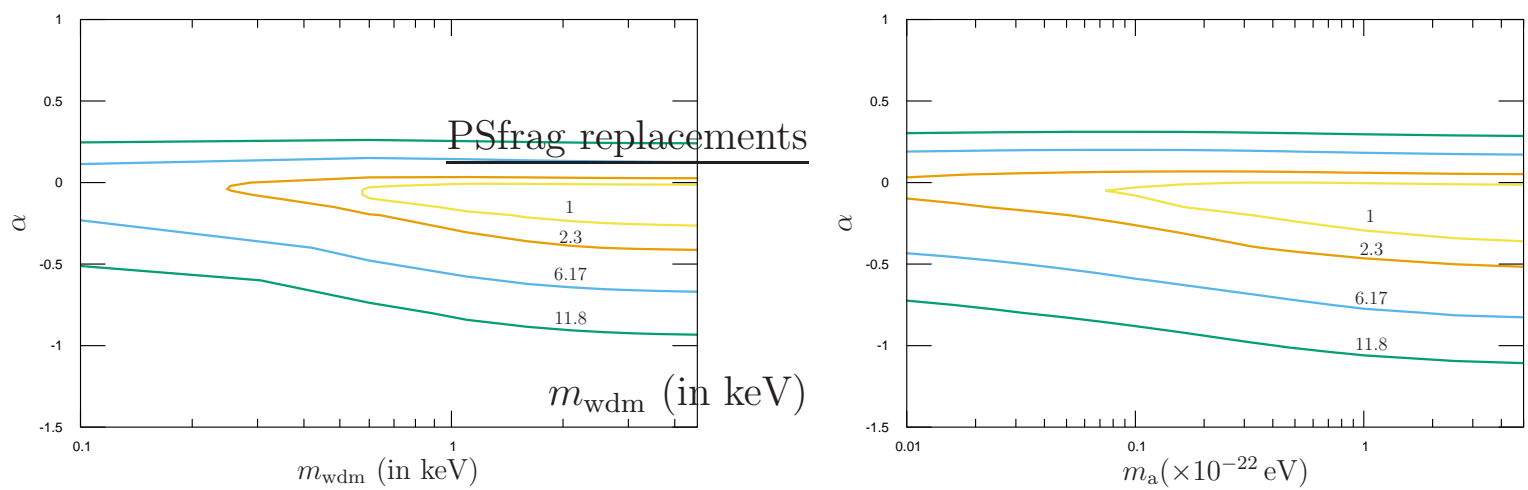

Figure 8. Figure shows contour plots for parameters: $m_{\mathrm{wdm}}$ and $\alpha$ for the WDM model (left panel), and $m_{\mathrm{a}}$ and $\alpha$ for the ULA model (right panel). The legends in the figures correspond to $\Delta \chi^{2}=1,2.3,6.17$ and 11.8 (from top to bottom).

consider two alternative dark matter models: ULA and WDM. Both these models yield lower matter power at small scales as compared to the $\Lambda$ CDM model.

We simulate the line-of-sight HI density field corresponding to Lyman- $\alpha$ forest and as our focus is the probe of small scales, the simulations resolve the Jeans' scale. Figure 4 allows us to gauge the impact of the alternative dark matter models of the evolution of effective optical depth. The effective optical depth for these model diverges from the prediction of the $\Lambda$ CDM model at higher redshifts. The percentage difference of these models from the $\Lambda$ CDM model nearly triples in the redshift range $4<z<6$. Therefore, data at higher redshifts is expected to be the principle discriminator between 

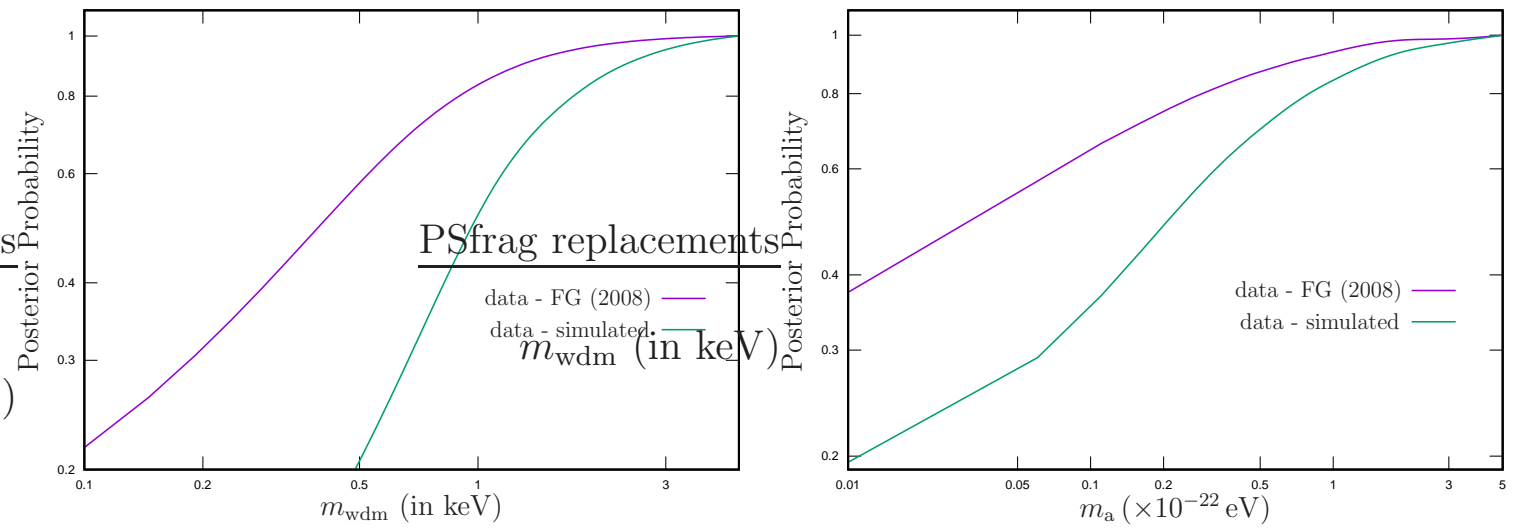

Figure 9. The Figure shows posterior probabilities of dark matter masses by comparing the theory with simulated data compatible with the $\Lambda$ CDM model. The posterior probabilities from Figure 7 are also shown (magenta curves) for comparison.

different dark matter models.

Using Likelihood analysis, we compare the evolution of the effective optical depth from the simulations with the high-resolution Lyman- $\alpha$ data [43] that spans a redshift range: $2 \leq z \leq 4.2$. The posterior probabilities of the dark matter masses are shown in Figure 7. These results yield the following $1-\sigma$ bounds on the dark matter masses: $m_{\mathrm{wdm}}>0.7 \mathrm{keV}$ and $m_{\mathrm{a}}>2 \times 10^{-23} \mathrm{eV}$ respectively for the WDM and the ULA models.

We next compare our theoretical predictions against simulated $\Lambda \mathrm{CDM}$ effective optical depth data sets over a larger redhift range: $2 \leq z \leq 6.5$. This allows us to assess the efficacy of high-redshift data as a discriminator between the different dark matter models (Figure 4). The resulting posterior probabilities are shown in Figure 9). The 1- $\sigma$ forecasts on the dark matter masses are more stringent in this case, as expected from the theory, and are given by: $m_{\mathrm{wdm}}>1.5 \mathrm{keV}$ and $m_{\mathrm{a}}>7 \times 10^{-23} \mathrm{eV}$.

The nature of dark matter remains a mystery. One possible way to understand its nature is to probe cosmological matter power spectrum at progressively smaller scales. The future detection of the 21-cm forest holds the promise of probing even smaller scales $([105])$.

\section{Acknowledgment}

AKS would like to thank RRI for allowing the access to the in-house cluster computing facility that was used for the computation needed for the analysis in this paper. AKS would also like to thank the anonymous referee(s) for useful suggestions and critical comments on our paper. KLP acknowledges the support of the Department of Atomic Energy, Government of India, under project no. RTI4001. 


\section{References}

[1] G. Hinshaw, D. Larson, E. Komatsu, D. N. Spergel, C. Bennett, J. Dunkley et al., Nine-year wilkinson microwave anisotropy probe (wmap) observations: cosmological parameter results, The Astrophysical Journal Supplement Series 208 (2013) 19.

[2] J. L. Sievers, R. A. Hlozek, M. R. Nolta, V. Acquaviva, G. E. Addison, P. A. Ade et al., The atacama cosmology telescope: Cosmological parameters from three seasons of data, Journal of Cosmology and Astroparticle Physics 2013 (2013) 060.

[3] N. Aghanim, Y. Akrami, M. Ashdown, J. Aumont, C. Baccigalupi, M. Ballardini et al., Planck 2018 results. vi. cosmological parameters, arXiv preprint arXiv:180\%.06209 (2018) .

[4] B. Abolfathi, D. Aguado, G. Aguilar, C. A. Prieto, A. Almeida, T. T. Ananna et al., The fourteenth data release of the sloan digital sky survey: First spectroscopic data from the extended baryon oscillation spectroscopic survey and from the second phase of the apache point observatory galactic evolution experiment, The Astrophysical Journal Supplement Series 235 (2018) 42.

[5] M. Hicken, W. M. Wood-Vasey, S. Blondin, P. Challis, S. Jha, P. L. Kelly et al., Improved dark energy constraints from 100 new cfa supernova type ia light curves, The Astrophysical Journal $\mathbf{7 0 0}$ (2009) 1097.

[6] A. Conley, J. Guy, M. Sullivan, N. Regnault, P. Astier, C. Balland et al., Supernova constraints and systematic uncertainties from the first three years of the supernova legacy survey, The Astrophysical Journal Supplement Series 192 (2010) 1.

[7] S. Ho, A. Cuesta, H.-J. Seo, R. De Putter, A. J. Ross, M. White et al., Clustering of sloan digital sky survey iii photometric luminous galaxies: the measurement, systematics, and cosmological implications, The Astrophysical Journal 761 (2012) 14.

[8] M. Bartelmann and P. Schneider, Weak gravitational lensing, Physics Reports 340 (2001) 291-472.

[9] N. Craig and A. Katz, The fraternal wimp miracle, Journal of Cosmology and Astroparticle Physics 2015 (2015) 054.

[10] G. Angloher, M. Bauer, I. Bavykina, A. Bento, C. Bucci, C. Ciemniak et al., Results from $730 \mathrm{~kg}$ days of the cresst-ii dark matter search, The European Physical Journal C 72 (2012) 1971.

[11] E. Aprile, K. Arisaka, F. Arneodo, A. Askin, L. Baudis, A. Behrens et al., First dark matter results from the xenon100 experiment, Physical Review Letters 105 (2010) 131302.

[12] Z. Ahmed, D. Akerib, S. Arrenberg, C. Bailey, D. Balakishiyeva, L. Baudis et al., Results from a low-energy analysis of the cdms ii germanium data, Physical Review Letters 106 (2011) 131302.

[13] D. S. Akerib, H. Araújo, X. Bai, A. Bailey, J. Balajthy, S. Bedikian et al., First results from the lux dark matter experiment at the sanford underground research facility, Physical review letters 112 (2014) 091303. 
[14] O. Adriani, G. Barbarino, G. Bazilevskaya, R. Bellotti, M. Boezio, E. Bogomolov et al., Pamela results on the cosmic-ray antiproton flux from 60 mev to 180 gev in kinetic energy, Physical Review Letters 105 (2010) 121101.

[15] M. Ackermann, M. Ajello, A. Allafort, W. Atwood, L. Baldini, G. Barbiellini et al., Measurement of separate cosmic-ray electron and positron spectra with the fermi large area telescope, Physical Review Letters 108 (2012) 011103.

[16] M. Aguilar, J. Alcaraz, J. Allaby, B. Alpat, G. Ambrosi, H. Anderhub et al., Cosmic-ray positron fraction measurement from 1 to $30 \mathrm{gev}$ with ams-01, Physics Letters B 646 (2007) 145-154.

[17] J. Goodman, M. Ibe, A. Rajaraman, W. Shepherd, T. M. Tait and H.-B. Yu, Constraints on light majorana dark matter from colliders, Physics Letters B 695 (2011) 185-188.

[18] P. J. Fox, R. Harnik, J. Kopp and Y. Tsai, Missing energy signatures of dark matter at the lhc, Physical Review D 85 (2012) 056011.

[19] B. Moore, S. Ghigna, F. Governato, G. Lake, T. Quinn, J. Stadel et al., Dark matter substructure within galactic halos, The Astrophysical Journal Letters 524 (1999) L19.

[20] A. Klypin, A. V. Kravtsov, O. Valenzuela and F. Prada, Where are the missing galactic satellites?, The Astrophysical Journal 522 (1999) 82.

[21] P. Peebles and A. Nusser, Nearby galaxies as pointers to a better theory of cosmic evolution, Nature 465 (2010) 565-569.

[22] J. Diemand, M. Kuhlen and P. Madau, Formation and evolution of galaxy dark matter halos and their substructure, The Astrophysical Journal 667 (2007) 859.

[23] J. F. Navarro, C. S. Frenk and S. D. White, A universal density profile from hierarchical clustering, The Astrophysical Journal 490 (1997) 493.

[24] J. Stadel, D. Potter, B. Moore, J. Diemand, P. Madau, M. Zemp et al., Quantifying the heart of darkness with ghalo-a multibillion particle simulation of a galactic halo, Monthly Notices of the Royal Astronomical Society: Letters 398 (2009) L21-L25.

[25] S. Garrison-Kimmel, M. Boylan-Kolchin, J. S. Bullock and E. N. Kirby, Too big to fail in the local group, Monthly Notices of the Royal Astronomical Society 444 (2014) 222-236.

[26] M. Boylan-Kolchin, J. S. Bullock and M. Kaplinghat, Too big to fail? the puzzling darkness of massive milky way subhaloes, Monthly Notices of the Royal Astronomical Society: Letters $4 \mathbf{1 5}$ (2011) L40-L44.

[27] R. A. Croft, D. H. Weinberg, N. Katz and L. Hernquist, Recovery of the power spectrum of mass fluctuations from observations of the lya forest, The Astrophysical Journal 495 (1998) 44.

[28] R. A. Croft, D. H. Weinberg, M. Pettini, L. Hernquist and N. Katz, The power spectrum of mass fluctuations measured from the lya forest at redshift $z=2.5$, The Astrophysical Journal 520 (1999) 1.

[29] R. A. Croft, D. H. Weinberg, M. Bolte, S. Burles, L. Hernquist, N. Katz et al., Toward a precise measurement of matter clustering: Lyo forest data at redshifts 2-4, The Astrophysical Journal 581 (2002) 20. 
[30] P. Mcdonald, R. Cen, D. H. Weinberg, S. Burles, D. P. Schneider, D. J. Schlegel et al., The linear theory power spectrum from the lyman- $\alpha$ forest in the sloan digital sky survey," astrophys, in J. 635, Citeseer.

[31] M. Rauch, The lyman alpha forest in the spectra of quasistellar objects, Annual Review of Astronomy and Astrophysics 36 (1998) 267-316.

[32] R. Mandelbaum, P. McDonald, U. Seljak and R. Cen, Precision cosmology from the lyman a forest: power spectrum and bispectrum, Monthly Notices of the Royal Astronomical Society 344 (2003) 776-788.

[33] M. Viel, S. Matarrese, A. Heavens, M. Haehnelt, T.-S. Kim, V. Springel et al., The bispectrum of the lyman $\alpha$ forest at $z ;$ 2-2.4 from a large sample of uves qso absorption spectra (luqas), Monthly Notices of the Royal Astronomical Society $\mathbf{3 4 7}$ (2004) L26-L30.

[34] L. Hernquist, N. Katz, D. H. Weinberg and J. Miralda-Escude, The lyman-alpha forest in the cold dark matter model, The Astrophysical Journal Letters 457 (1996) L51.

[35] P. McDonald and J. Miralda-Escudé, Measuring the cosmological geometry from the lya forest along parallel lines of sight, The Astrophysical Journal 518 (1999) 24.

[36] J. Lesgourgues, R. Massey, M. Viel and M. Hähnelt, A combined analysis of lyman-alpha forest, 3d weak lensing and wmap year three data, tech. rep., 2007.

[37] R. A. Croft, W. Hu and R. Dave, Cosmological limits on the neutrino mass from the ly a forest, Physical Review Letters 83 (1999) 1092.

[38] C. Yèche, N. Palanque-Delabrouille, J. Baur and H. D. M. Des Bourboux, Constraints on neutrino masses from lyman-alpha forest power spectrum with boss and xq-100, Journal of Cosmology and Astroparticle Physics 2017 (2017) 047.

[39] P. McDonald and D. J. Eisenstein, Dark energy and curvature from a future baryonic acoustic oscillation survey using the lyman- $\alpha$ forest, Physical Review D 76 (2007) 063009 .

[40] A. Slosar, V. Iršič, D. Kirkby, S. Bailey, T. Delubac, J. Rich et al., Measurement of baryon acoustic oscillations in the lyman- $\alpha$ forest fluctuations in boss data release 9, Journal of Cosmology and Astroparticle Physics 2013 (2013) 026.

[41] T. Delubac, J. Rich, S. Bailey, A. Font-Ribera, D. Kirkby, J.-M. Le Goff et al., Baryon acoustic oscillations in the lya forest of boss quasars, Astronomy 8 Astrophysics 552 (2013) A96.

[42] T. Delubac, J. E. Bautista, J. Rich, D. Kirkby, S. Bailey, A. Font-Ribera et al., Baryon acoustic oscillations in the lya forest of boss dr11 quasars, Astronomy 8 Astrophysics $\mathbf{5 7 4}$ (2015) A59.

[43] C.-A. Faucher-Giguere, J. X. Prochaska, A. Lidz, L. Hernquist and M. Zaldarriaga, A direct precision measurement of the intergalactic lya opacity at $2 \leq z \leq 4.2$, The Astrophysical Journal 681 (2008) 831.

[44] J. S. Bolton, S. P. Oh and S. R. Furlanetto, The evolution of the ly $\alpha$ forest effective optical depth following he ii reionization, Monthly Notices of the Royal Astronomical Society 396 (2009) 2405-2418. 
[45] G. D. Becker, P. C. Hewett, G. Worseck and J. X. Prochaska, A refined measurement of the mean transmitted flux in the ly- $\alpha$ forest over $2<z<5$ using composite quasar spectra, Monthly Notices of the Royal Astronomical Society 430 (2013) 2067-2081.

[46] V. Kamble, K. Dawson, H. d. M. des Bourboux, J. Bautista and D. P. Scheinder, Measurements of effective optical depth in the lya forest from the boss dr12 quasar sample, The Astrophysical Journal 892 (2020) 70.

[47] H. Bi and A. F. Davidsen, Evolution of structure in the intergalactic medium and the nature of the lya forest, The Astrophysical Journal 479 (1997) 523.

[48] K. L. Pandey and S. K. Sethi, Probing primordial magnetic fields using lya clouds, The Astrophysical Journal 762 (2012) 15.

[49] L. Zhang, J. Redondo and G. Sigl, Galactic signatures of decaying dark matter, Journal of Cosmology and Astroparticle Physics 2009 (2009) 012.

[50] A. Boyarsky, D. Iakubovskyi, O. Ruchayskiy and V. Savchenko, Constraints on decaying dark matter from xmm-newton observations of m31, Monthly Notices of the Royal Astronomical Society 387 (2008) 1361-1373.

[51] A. Boyarsky, J. Lesgourgues, O. Ruchayskiy and M. Viel, Realistic sterile neutrino dark matter with kev mass does not contradict cosmological bounds, Physical review letters 102 (2009) 201304.

[52] U. Seljak, A. Makarov, P. McDonald and H. Trac, Can sterile neutrinos be the dark matter?, Physical Review Letters 97 (2006) 191303.

[53] R. E. Smith and K. Markovic, Testing the warm dark matter paradigm with large-scale structures, Physical Review D 84 (2011) 063507.

[54] J. Ellis, J. Hagelin, D. Nanopoulos, K. Olive and M. Srednicki, Supersymmetric relics from the big bang, 1984.

[55] S. Dodelson and L. M. Widrow, Sterile neutrinos as dark matter, Physical Review Letters 72 (1994) 17.

[56] A. Arvanitaki, S. Dimopoulos, S. Dubovsky, N. Kaloper and J. March-Russell, String axiverse, Physical Review D 81 (2010) 123530.

[57] J. A. Frieman, C. T. Hill, A. Stebbins and I. Waga, Cosmology with ultralight pseudo nambu-goldstone bosons, Physical Review Letters 75 (1995) 2077.

[58] K. Coble, S. Dodelson and J. A. Frieman, Dynamical $\lambda$ models of structure formation, Physical Review D 55 (1997) 1851.

[59] W. Hu, R. Barkana and A. Gruzinov, Fuzzy cold dark matter: the wave properties of ultralight particles, Physical Review Letters 85 (2000) 1158.

[60] D. J. Marsh and P. G. Ferreira, Ultralight scalar fields and the growth of structure in the universe, Physical Review D $\mathbf{8 2}$ (2010) 103528.

[61] C.-G. Park, J.-c. Hwang and H. Noh, Axion as a cold dark matter candidate: low-mass case, Physical Review D 86 (2012) 083535.

[62] T. Kobayashi, R. Murgia, A. De Simone, V. Iršič and M. Viel, Lyman- $\alpha$ constraints on ultralight scalar dark matter: Implications for the early and late universe, Physical Review D 96 (2017) 123514. 
[63] M. Viel, G. D. Becker, J. S. Bolton and M. G. Haehnelt, Warm dark matter as a solution to the small scale crisis: New constraints from high redshift lyman- $\alpha$ forest data, Physical Review D $8 \mathbf{8}$ (2013) 043502.

[64] E. Polisensky and M. Ricotti, Constraints on the dark matter particle mass from the number of milky way satellites, Physical Review D 83 (2011) 043506.

[65] D. Anderhalden, J. Diemand, G. Bertone, A. V. Maccio and A. Schneider, The galactic halo in mixed dark matter cosmologies, Journal of Cosmology and Astroparticle Physics 2012 (2012) 047.

[66] M. R. Lovell, V. Eke, C. S. Frenk, L. Gao, A. Jenkins, T. Theuns et al., The haloes of bright satellite galaxies in a warm dark matter universe, Monthly Notices of the Royal Astronomical Society 420 (2012) 2318-2324.

[67] A. V. Macciò, S. Paduroiu, D. Anderhalden, A. Schneider and B. Moore, Cores in warm dark matter haloes: a catch 22 problem, Monthly Notices of the Royal Astronomical Society 424 (2012) 1105-1112.

[68] A. Schneider, R. E. Smith, A. V. Macciò and B. Moore, Non-linear evolution of cosmological structures in warm dark matter models, Monthly Notices of the Royal Astronomical Society 424 (2012) 684-698.

[69] J. Baur, N. Palanque-Delabrouille, C. Yèche, C. Magneville and M. Viel, Lyman-alpha forests cool warm dark matter, Journal of Cosmology and Astroparticle Physics 2016 (2016) 012.

[70] D. J. Marsh and A.-R. Pop, Axion dark matter, solitons and the cusp-core problem, Monthly Notices of the Royal Astronomical Society 451 (2015) 2479-2492.

[71] D. J. Marsh, Axion cosmology, Physics Reports 643 (2016) 1-79.

[72] L. Hui, J. P. Ostriker, S. Tremaine and E. Witten, On the hypothesis that cosmological dark matter is composed of ultra-light bosons, arXiv preprint arXiv:1610.08297 (2016) .

[73] A. Sarkar, R. Mondal, S. Das, S. K. Sethi, S. Bharadwaj and D. J. Marsh, The effects of the small-scale dm power on the cosmological neutral hydrogen (hi) distribution at high redshifts, Journal of Cosmology and Astroparticle Physics 2016 (2016) 012.

[74] A. Sarkar, S. K. Sethi and S. Das, The effects of the small-scale behaviour of dark matter power spectrum on cmb spectral distortion, Journal of Cosmology and Astroparticle Physics 2017 (2017) 012.

[75] R. Hlozek, D. Grin, D. J. Marsh and P. G. Ferreira, A search for ultralight axions using precision cosmological data, Physical Review D 91 (2015) 103512.

[76] K. K. Rogers and H. V. Peiris, Strong bound on canonical ultra-light axion dark matter from the lyman-alpha forest, arXiv preprint arXiv:200\%.12705 (2020) .

[77] N. Palanque-Delabrouille, C. Yèche, A. Borde, J.-M. Le Goff, G. Rossi, M. Viel et al.,

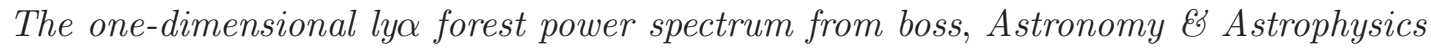
559 (2013) A85.

[78] V. Iršič, M. Viel, T. A. Berg, V. D’Odorico, M. G. Haehnelt, S. Cristiani et al., The lyman $\alpha$ forest power spectrum from the $x q-100$ legacy survey, Monthly Notices of the Royal Astronomical Society 466 (2017) 4332-4345. 
[79] S. Chabanier, N. Palanque-Delabrouille, C. Yèche, J.-M. Le Goff, E. Armengaud, J. Bautista et al., The one-dimensional power spectrum from the sdss dr14 lya forests, Journal of Cosmology and Astroparticle Physics 2019 (2019) 017.

[80] J. R. Bond, G. Efstathiou and J. Silk, Massive neutrinos and the large-scale structure of the universe, Physical Review Letters 45 (1980).

[81] M. Viel, J. Lesgourgues, M. G. Haehnelt, S. Matarrese and A. Riotto, Constraining warm dark matter candidates including sterile neutrinos and light gravitinos with wmap and the lyman- $\alpha$ forest, Physical Review D 71 (2005) 063534.

[82] R. E. Smith and K. Markovic, Testing the warm dark matter paradigm with large-scale structures, Physical Review D 84 (2011) 063507.

[83] M. S. Seigar, Cold dark matter, hot dark matter, and their alternatives, in Dark Matter in the Universe. Morgan \& Claypool Publishers, 2015.

[84] X. Kang, Warm dark matter model with a few kev mass is bad for the too-big-to-fail problem, Monthly Notices of the Royal Astronomical Society 491 (2020) 2520-2535.

[85] A. R. Zentner and J. S. Bullock, Halo substructure and the power spectrum, The Astrophysical Journal 598 (2003) 49.

[86] P. Bode, J. P. Ostriker and N. Turok, Halo formation in warm dark matter models, The Astrophysical Journal 556 (2001) 93.

[87] K. Markovic, S. Bridle, A. Slosar and J. Weller, Constraining warm dark matter with cosmic shear power spectra, Journal of Cosmology and Astroparticle Physics 2011 (2011) 022.

[88] A. Arvanitaki, S. Dimopoulos, S. Dubovsky, N. Kaloper and J. March-Russell, String axiverse, Physical Review D 81 (2010) 123530.

[89] D. J. Marsh and P. G. Ferreira, Ultralight scalar fields and the growth of structure in the universe, Physical Review D 82 (2010) 103528.

[90] W. Hu, R. Barkana and A. Gruzinov, Fuzzy cold dark matter: the wave properties of ultralight particles, Physical Review Letters 85 (2000) 1158.

[91] L. Amendola and R. Barbieri, Dark matter from an ultra-light pseudo-goldsone-boson, Physics Letters B 642 (2006) 192-196.

[92] T. R. Choudhury, R. Srianand and T. Padmanabhan, Semianalytic approach to understanding the distribution of neutral hydrogen in the universe: comparison of simulations with observations, The Astrophysical Journal 559 (2001) 29.

[93] L. Hui and N. Y. Gnedin, Equation of state of the photoionized intergalactic medium, Monthly Notices of the Royal Astronomical Society 292 (1997) 27-42.

[94] J. X. Prochaska and A. M. Wolfe, Chemical abundances of the damped lyo systems at $z_{\dot{z}}$ 1.5, The Astrophysical Journal Supplement Series 121 (1999) 369.

[95] J. X. Prochaska, A. M. Wolfe, J. C. Howk, E. Gawiser, S. M. Burles and J. Cooke, The ucsd/keck damped lya abundance database: a decade of high-resolution spectroscopy, The Astrophysical Journal Supplement Series 171 (2007) 29. 
[96] J. M. O'meara, J. X. Prochaska, S. Burles, G. Prochter, R. A. Bernstein and K. M. Burgess, The keck+ magellan survey for lyman limit absorption. $i$. the frequency distribution of super lyman limit systems, The Astrophysical Journal 656 (2007) 666.

[97] D. P. Schneider, G. T. Richards, P. B. Hall, M. A. Strauss, S. F. Anderson, T. A. Boroson et al., The sloan digital sky survey quasar catalog. v. seventh data release, The Astronomical Journal 139 (2010) 2360.

[98] M. McQuinn, The evolution of the intergalactic medium, Annual Review of Astronomy and Astrophysics 54 (2016) 313-362.

[99] P. La Plante, H. Trac, R. Croft and R. Cen, Helium reionization simulations. iii. the helium lya forest, The Astrophysical Journal 868 (2018) 106.

[100] B. T. Draine, Physics of the interstellar and intergalactic medium, vol. 19. Princeton University Press, 2010.

[101] Z. Lukić, C. W. Stark, P. Nugent, M. White, A. A. Meiksin and A. Almgren, The lyman $\alpha$ forest in optically thin hydrodynamical simulations, Monthly Notices of the Royal Astronomical Society 446 (2015) 3697-3724.

[102] C.-A. Faucher-Giguere, A. Lidz, L. Hernquist and M. Zaldarriaga, A flat photoionization rate at $2 \leq z \leq 4.2$ : Evidence for a stellar-dominated uv background and against a decline of cosmic star formation beyond $z$ 3, The Astrophysical Journal Letters 682 (2008) L9.

[103] M. Viel, M. G. Haehnelt and V. Springel, Inferring the dark matter power spectrum from the lyman a forest in high-resolution qso absorption spectra, Monthly Notices of the Royal Astronomical Society 354 (2004) 684-694.

[104] M. Viel, The lyman- $\alpha$ forest as a probe of the coldness of dark matter, in IFAE 200\%, pp. 255-260. Springer, 2008.

[105] H. Shimabukuro, K. Ichiki and K. Kadota, Constraining the nature of ultra light dark matter particles with the $21 \mathrm{~cm}$ forest, Physical Review D 101 (2020) 043516. 